\title{
Lampbrush chromosomes enable study of cohesin dynamics
}

\author{
Christopher Austin • Natalya Novikova • \\ Vincent Guacci • Michel Bellini
}

Published online: 20 March 2009

(C) Springer Science + Business Media B.V. 2009

\begin{abstract}
The lampbrush chromosomes present in the nuclei of amphibian oocytes offer unique biological approaches for study of the mechanisms that regulate chromatin structure with high spatial resolution. We discuss fundamental aspects of the remarkable organization and plasticity exhibited by lampbrush chromosomes. We then utilize lampbrush chromosomes to characterize the chromosomal distribution and dynamics of cohesin, the four-protein complex (RAD21/MCD1/ $\mathrm{SCC} 1, \mathrm{SMC} 1, \mathrm{SMC} 3, \mathrm{SCC} 3 / \mathrm{SA} 2)$ responsible for sister chromatid cohesion. We find that endogenous SMC3 and newly expressed hRAD21 co-localize on chromosomal axes, sites where sister chromatids are tightly paired. We present evidence suggesting that hRAD21 recruitment to lampbrush chromosomes is modulated by chromosomal SMC1 and SMC3. Notably, using a tech-
\end{abstract}

Responsible Editor: Dr. Christian Haering.

C. Austin $\cdot$ N. Novikova $\cdot$ M. Bellini

Cell \& Developmental Biology,

University of Illinois at Urbana-Champaign,

Urbana, IL, USA

V. Guacci

Embryology, Carnegie Institution of Washington,

Baltimore, MD, USA

M. Bellini $(\square)$

Department of Cell and Developmental Biology,

School of Molecular and Cellular Biology,

601 South Goodwin Avenue, Room B107 CLSL,

Urbana, IL 61801, USA

e-mail: bellini@life.uiuc.edu nique for de novo chromosome assembly, we demonstrate that both SMC3 and hRAD21 are recruited to single, unreplicated lampbrush chromatids. Finally, we used our novel method of analyzing the oocyte nucleus under oil combined with fluorescence recovery after photobleaching, to provide direct evidence that cohesin is highly dynamic at discrete, condensed chromosomal regions. Collectively, these data demonstrate that lampbrush chromosomes provide a unique and powerful tool for combining biochemical and cytological analyses for dissection of complex chromosomal processes.

Keywords lampbrush chromosome - chromatin . cohesin $\cdot$ SMC $\cdot$ oocyte nucleus

$\begin{array}{ll}\text { Abbreviations } \\ \text { EGFP } & \text { enhanced green fluorescent protein } \\ \text { FRAP } & \text { fluorescence recovery after photobleaching } \\ \text { LBC } & \text { lampbrush chromosome } \\ \text { SMC } & \text { structural maintenance of chromosomes } \\ \text { RNP } & \text { ribonucleoprotein } \\ \text { RNAPII } & \text { RNA polymerase II } \\ \text { YFP } & \text { yellow fluorescent protein }\end{array}$

\section{Introduction}

In the nucleus of a Xenopus laevis oocyte, chromatin is organized into 18 lampbrush chromosomes (LBCs) and approximately 2000 extrachromosomal nucleoli, 
which form around amplified copies of the rDNA genes. LBCs were reported for the first time in amphibians (Flemming 1882) and were subsequently demonstrated to be present in the oocytes of most animals. These chromosomes earned the name 'lampbrush' when they were compared with oil-lamp brushes (Rückert 1892) for the striking resemblance of their respective structural organization, i.e., a central axis from which numerous lateral bristles project radially. This excellent analogy has been used since then, as it provides a simple way to represent LBCs three-dimensionally. However, it does little to emphasize the giant size of LBCs, which is the major reason why they were observed by light microscopy more than a century ago. Remarkably, some LBCs in growing oocytes can measure near one millimeter in length. This highly extended morphology is directly correlated with their intense transcriptional activity, which is needed to sustain the growth of the oocyte and the storage of many transcripts and proteins for use during early development. As the oocyte approaches maturity, transcriptional activity decreases, and LBCs undergo morphological changes to eventually achieve a more compact structure typical of meiotic metaphase bivalents. Therefore, LBCs provide a window of opportunity to observe detailed chromosomal features otherwise irresolvable at the light microscopy level.

An excellent review of the complex structural and functional organization of LBCs, which emphasizes their unique advantageous properties for studying RNA transcription and processing, is already available (Morgan 2002). Here we describe the main traits of LBCs that make them particularly amenable to the cytological study of meiotic chromatin. We also present original data on the chromosomal distribution and dynamics of two members of the cohesin complex. These studies demonstrate the unique and powerful system that LBCs and the amphibian oocyte provide for investigation of fundamental questions in chromosome biology described in this special issue.

The LBC architecture

LBCs are diplotene bivalent chromosomes, with homologues still attached to each other at chiasmata. The axis of each homologue consists of two sister chromatids tightly paired (cohesed) and organized in a linear arrangement of compact chromatin granules (chromomeres), which are interspaced by regions of very thin chromatin fiber (Fig. 1A and B). The many chromomeres support lateral projections of highly decondensed chromatin loops, which result in the characteristic appearance of the LBCs. While, the loops are the most conspicuous trait of LBCs, they represent only a very small fraction of the chromosomal DNA (Leon and Kezer 1990). These lateral loops correspond to domains of unusually intense transcriptional activity. Accordingly, RNA polymerase II (RNAPII) was demonstrated to be present at a high density, actively engaged in transcription, over the length of each loop chromatin axis (Gall et al. 1999 and Fig. 1C). As a result, the axis is surrounded by a thick ribonucleoprotein (RNP) matrix, which is composed of the many elongating transcripts packed together with an ensemble of processing factors into RNP fibrils. The very high number of active elongation complexes on the axis of a loop does not prevent the association of histones, however. In good agreement with the intense transcriptional activity associated with these regions, histone H4 was found to be acetylated on different lysine residues (Sommerville et al. 1993). Strikingly, newly expressed histones, such as H4, were shown by Dr. J.G. Gall to associate with loop axes primarily at places where RNAPII is absent (Fig. 1C), which permits one to speculate that the chromatin of a loop is less compacted than a $10 \mathrm{~nm}$ fiber. Consequently, the fact that a lateral loop is readily distinguishable by phase-contrast microscopy is not due to its chromatin component, but rather to the high density of nascent RNP fibrils surrounding it. Indeed, the loops corresponding to RNAPIII transcription units are readily detected by immunofluorescence using anti-RNAPIII antibodies, but are not visible by phase-contrast microscopy as they lack an RNP matrix (Murphy et al. 2002). Importantly, the lateral loops correspond to regions where, unlike in chromomeres, the sister chromatids are separated physically, hence their occurrence in homologous pairs.

\section{The LBC chromatin}

Several of the previous studies aiming at characterizing LBCs revealed that they lack some of the typical chromatin components (reviewed in Morgan 2002). A number of the differences can be directly accounted by the intense transcriptional activity of LBCs. For instance, the histone $\mathrm{H} 1$, which is implicated in the 


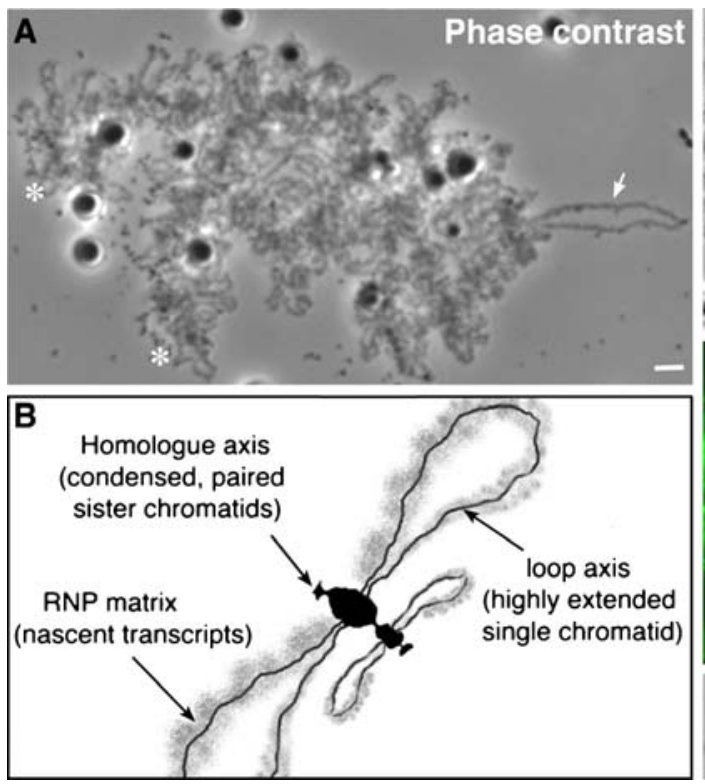

Fig. 1 The lampbrush chromosome. (A) Phase-contrast image showing one of the $18 \mathrm{LBCs}$ present in the nucleus of a Xenopus laevis oocyte. The two homologues are indicated by asterisks. An arrow indicates a particularly well-extended lateral loop. Scale bar represents $5 \mu \mathrm{m}$. (B) Schematic representation of a small region of one of the two homologues forming an LBC. The chromosomal axis is composed of a linear array of compacted chromatin granules (chromomeres), from which arise pairs of chromatin loops surrounded by nascent RNP fibrils. (C) The extended chromatin of the lateral loops. A phase-contrast
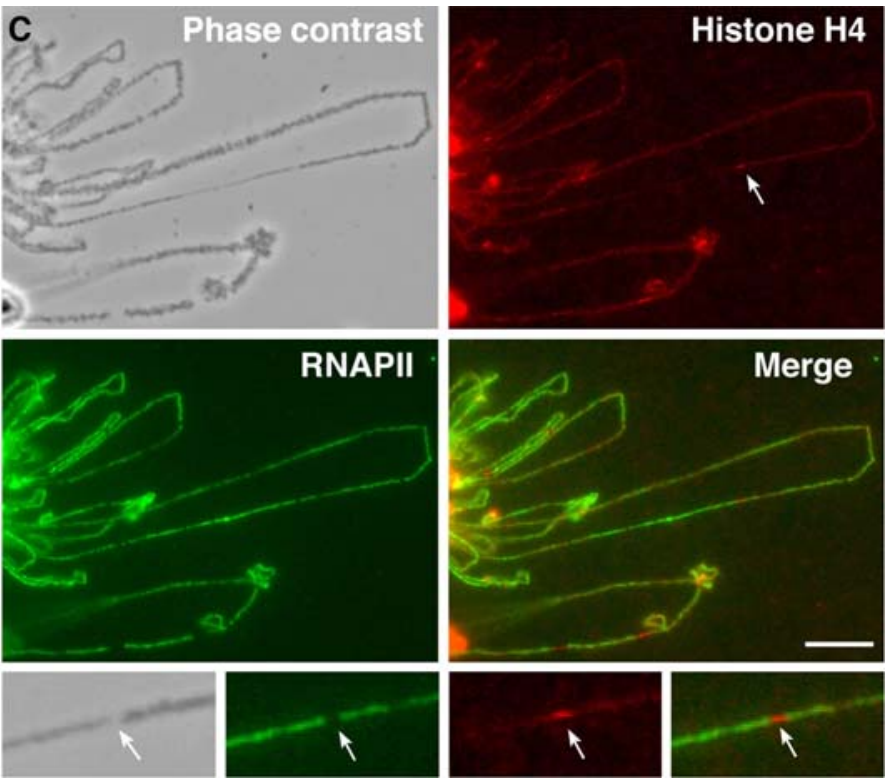

micrograph and its corresponding fluorescent images showing the distribution of a newly expressed HA-tagged histone H4 (red) and endogenous RNAPII (green) on several loops of a newt LBC. Both proteins are present over the entire length of the loops but in strikingly discontinuous patterns. Remarkably, H4 appears to accumulate where RNAPII is missing (see the magnified view of a small region of a loop indicated by an arrow). These images were kindly provided by Dr. J.G. Gall (Carnegie Institution of Washington). Scale bar represents $10 \mu \mathrm{m}$

made in comparison with the greatly uncompacted lateral loops. In fact, the inherent extended nature of LBCs and their subsequent dramatic condensation during oocyte maturation clearly indicate that chromomeres are considerably less compacted than mitotic chromosomes. Thus, many factors required to achieve a high level of chromatin compaction during mitosis, may not be necessary for the overall LBC architecture. Despite their apparent differences in composition, both the LBCs and somatic chromosomes represent a highly dynamic environment for chromatin-associated factors. This property was illustrated for LBCs in a recent study, where LBCassociated histone $\mathrm{H} 3$ was shown to exchange rapidly and continuously with the free nucleoplasmic pool of H3 (Stewart et al. 2006). In agreement with this remarkable finding is the fact that newly expressed histone $\mathrm{H} 4$ also rapidly targets the loops and axes of LBCs (Joseph Gall, unpublished data generously provided for Fig. 1). 
Cohesin and sister chromatin cohesion

The establishment, maintenance, and dissolution of sister chromatid cohesion are critical events that must be precisely regulated to ensure the faithful segregation of chromosomes during mitosis and meiosis. Initial work from yeast identified a 'cohesin' complex comprising four proteins, MCD1 (also called SCC1), $\mathrm{SMC} 1, \mathrm{SMC} 3$, and SCC3, which functions as a molecular glue required for sister chromatid cohesion during the mitotic cell cycle (Guacci et al. 1997; Michaelis et al. 1997; Toth et al. 1999). Cohesin is evolutionarily conserved in eukaryotes (reviewed in Guacci 2007; Onn et al. 2008). Xenopus laevis cohesin was characterized in egg extracts and shown to localize between sister chromatids of condensed mitotic chromosomes (Losada et al. 1998, 2000). Unlike in yeast, there are two forms of cohesin in somatic cells. Both contain three common cohesin subunits, MCD1/RAD21 (also called SCC1), SMC1, and $\mathrm{SMC} 3$, but differ in that they contain either one of two SCC3 isoforms, called SA1 or SA2 (Losada et al. 2000; Sumara et al. 2000). Xenopus cohesin was also shown to mediate cohesion, since depletion of cohesin from egg extracts causes defects in sister chromatid cohesion (Losada et al. 1998).

There is abundant evidence supporting the conclusion that SMC1 and SMC3 bind to each other via their central hinge domains to form heterodimers, which dictate the overall structure of cohesin (Haering et al. 2002; Jessberger 2002, 2003). Data from yeast indicate that MCD1/RAD21 interacts with the SMC subunits through its amino- and carboxy-terminal ends to form a tripartite ring-like structure (Gruber et al. 2003; Haering et al. 2002). The MCD1/RAD21 subunit also provides the binding domain for SCC3 (Gruber et al. 2003; Haering et al. 2002). MCD1/ RAD21 is cleaved by a protease called separase (Ciosk et al. 1998; Kumada et al. 1998; Uhlmann et al. 1999; Waizenegger et al. 2000), which is activated by the anaphase-promoting complex (APC)-mediated destruction of its inhibitor Pds1p/securin (Cohen-Fix et al. 1996; Funabiki et al. 1996). This process is evolutionary conserved. Finally, MCD1/RAD21 is also phosphorylated by Polo kinase, which is important for cohesion dissolution in budding yeast (Alexandru et al. 2001). Together, these data indicate that the MCD1/RAD21 is a key regulator for cohesin function.
Cohesin and meiosis

Chromosome segregation during meiosis is more complex than during mitosis. The reason is that sister chromatids as well as homologues must be held together during meiosis I, yet homologues must disjoin while sisters remain cohesed during anaphase I. Part of this differential regulation is accomplished by the formation of meiotic cohesin complexes, in which single subunits are replaced by meiosis-specific isoforms. A substitution that is conserved among eukaryotes is the replacement of MCD1/RAD21 by REC8. The REC8 meiotic cohesin has essential roles in the formation of the synaptonemal complex, sister chromatid cohesion, and homologous recombination in yeast (reviewed in Stoop-Myer and Amon 1999; Revenkova and Jessberger 2005; Brar et al. 2006). Mammalian orthologues of REC8 exist (McKay et al. 1996; Parisi et al. 1999) and were shown to also play critical roles during meiosis (reviewed in Revenkova and Jessberger 2005). Cohesin complexes containing meiosis-specific isoforms of SMC1 (called SMC1B) and SCC3 (called STAG3) have also been studied in mice.

The MCD1/RAD21-containing complexes are termed mitotic cohesin, in part because they were initially found in cells undergoing the mitotic cell cycle. The term mitotic cohesin is a bit of a misnomer, as this form of cohesin is clearly present during meiosis and plays a major role in meiotic chromosome structure and segregation. This conclusion initially came from mammalian cells, where RAD21 was demonstrated to be associated with mammalian meiotic chromosomes in spatial and temporal patterns that strongly suggest a role in the formation of the synaptonemal complex and sister chromatin cohesion (Parra et al. 2004; Prieto et al. 2004; Xu et al. 2004; $\mathrm{Xu}$ et al. 2005). Early data from budding yeast suggested that MCD1 had little or no meiotic role, as very little mitotic cohesin is present but meiotic cohesin is highly expressed (Klein et al. 1999). These results set up a situation in which yeast and vertebrates were fundamentally different in meiosis. However, subsequent data from budding yeast showed that small amounts of mitotic cohesin are present and essential for proper meiotic chromosome segregation (Kateneva et al. 2005). Thus, mitotic cohesin plays important roles during meiosis in all eukaryotes, but the ratio of mitotic/meiotic cohesins 
varies among organisms. It may be that the higher amounts of mitotic cohesin present in vertebrate meiosis, as compared with yeast meiosis, reflect the much larger size of vertebrate chromosomes, and the consequent need for higher compaction to enable segregation.

Has cohesin a role in structuring LBCs?

LBCs represent a unique opportunity for the cytological analysis of factors involved in meiotic sister chromatid cohesion. Even though the overall axial organization of LBCs has not been precisely defined, the generally accepted model of LBC chromomeric configuration is that the two sister chromatids of a homologue are held together in close proximity at their bases to organize homologous loop domains of chromatin (reviewed in Morgan 2002; see Fig. 1B).

Such a principle of organization was previously postulated to explain a link between sister chromatid cohesion and mitotic chromosome condensation in budding yeast (Guacci et al. 1997). In this model, sites of sister chromatid cohesion are distributed at discrete loci along the chromosome length, which enables the chromatin between cohesion sites to form loops and thereby cause chromatin compaction. Studies using chromatin immunoprecipitation revealed that yeast cohesin is distributed at approximately $10 \mathrm{~kb}$ intervals along chromosomes (Glynn et al. 2004), consistent with cohesin being located at the base of the postulated chromatin loops.

Since the basic structure of an LBC resembles how yeast chromosomes might be organized by cohesin, one might expect that cohesin would also play an important role in LBC structure. However, initial work using avian LBC and cohesin antibodies reported only a weak association of cohesin along the length of LBC axes in a non-uniform pattern and limited to few inter-chromomeric sites. The only prominent sites of cohesin accumulation were at centromere protein bodies (PBs), which were proposed to be sites of storage for chromosomal factors until early embryogenesis (Krasikova et al. 2005, 2006).

Here, we use amphibian LBCs to provide a fine structural mapping of the chromosomal association of cohesins. In contrast to the initial reports on avian LBCs, we show that endogenous SMC3 and a newly expressed hRAD21 localize at high levels along the entire length of the LBC axes, where sister chromatids are tightly paired. We also find that cohesin staining is very robust at the axes, whereas little or no cohesin is found on loops. Finally, we utilized our novel under oil method of characterizing chromosomes of the Xenopus oocyte (Patel et al. 2008) to study cohesin dynamics by fluorescence recovery after photobleaching (FRAP) of YFP-hRAD21. These data are among the first direct evidence (see also McNairn and Gerton, this issue) for cohesin dynamics on a small and defined region of a single chromosome. Moreover, cohesin appears more dynamic than initially reported in vertebrate somatic cells.

\section{Materials and methods}

DNA cloning, in vitro transcription and protein expression

Three HA (hemagglutinin) tags (see below) were added in frame at the $3^{\prime}$ end of the open reading frame (ORF) coding for the human RAD21. The resulting hRAD21-3HA ORF was then cloned into the pcDNA 3.1 vector (Invitrogen, Carlsbad, CA, USA) between the HindIII and EcoRV restriction sites. All hRAD21 deletion mutants were obtained by polymerase chain reaction (PCR), using the high fidelity Deep Vent ${ }_{R}{ }^{\circledR}$ DNA Polymerase (New England BioLabs, Ipswich, MA, USA) and the hRAD21-3HA cDNA as a template. All received an SV40 NLS (CCA AAG AAG AAG CGA AAG CTG) in their $3^{\prime}$ end to ensure that the corresponding proteins would enter the nucleus. The following primers were used for the amino terminal deletions of hRAD21: $\triangle \mathrm{N} 126$ (5'GCC AGA ACA ATG ATC GAT GTG GCC CAG C and 3'-GCC AGT GTG ATG GAT GGG GAT CCG); $\triangle \mathrm{N} 197$ (5'-GCC AGA ACA ATG AGC ACC AGC AAT CTG AAT GAG and 3'-GCC AGT GTG ATG GAT GGG GAT CCG); $\triangle \mathrm{N} 288$ (5'-GCC AGA ACA ATG ACT GAT CAA ACA ACA C and 3'-GCC AGT GTG ATG GAT GGG GAT CCG); $\triangle \mathrm{N} 467$ (5'GCC AGA ACA ATG CCT CCA CCA CCA CCT CAG and 3'-CTA CAG CTT TCG CTT CTT CTT TGG CAG ATC TCC GCA CTG). The carboxyl deletion of hRAD21, $\triangle \mathrm{C} 523$, was produced in a twostep PCR procedure. In the first step, the hRAD21 ORF was used as DNA template and the following primers were used to amplify the region of interest: 5'-CTT CTG TTT TCA TCT CGA GCC AGA ACA ATG TT CTA C and 3'-ATA GTC AGG AAC ATC GTA TGG GTA 
CCATCT TCT TTC TTC CTG ATC. In the second step, the HA tag was completed and the SV40 NLS was added using the following primers: 5'-CTT CTG TTT TCA TCT CGA GCC AGA ACA ATG TT CTA C and 3'TCA CAG CTT TCG CTT CTT CTT TGG CGC ATA GTC AGG AAC ATC.

The amplified DNA fragments were cloned into the pCR ${ }^{\circledR}$-Blunt II-TOPO ${ }^{\circledR}$ vector (Invitrogen), which contains an SP6 and a T7 promoter localized upstream and downstream of the multiple cloning site, respectively. The YFP-hRAD21-3HA clone was produced in two steps. First, the hRAD21-3HA ORF was PCR-amplified and fused in frame downstream of the YFP ORF of the YFP-C1 vector (Clontech, Mountain View, CA, USA), between the XhoI and $K p n I$ restriction sites. The following primers were used: 5'-CTT CTG TTT TCA TCT CGA GCC AGA ACA ATG TT CTA $C$ and 3'-GTG ATG GAT GGG GTA CCG ATT TTA CAG ATC TCC. Second, the resulting YFP-hRAD21-3HA ORF was amplified by PCR and cloned into the $\mathrm{pCR}^{\circledR}$-Blunt II-TOPO ${ }^{\circledR}$ vector (Invitrogen). The following primers were used: 5'-CGA ATT AAC CCT CAC TAA AGG GAT TTC CAA GTC TCC ACC and 3'-TGT GGT ATG GCT GAT TAT GAT C. Finally, the YFP- $\triangle$ C125 DNA was amplified by from the YFP-hRAD21-3HA template, using the following primers: 5'-CGA ATT AAC CCT CAC TAA AGG GAT TTC CAA GTC TCC ACC and 3'-ATA GTC AGG AAC ATC GTA TGG GTA GTC ATC TAA GTC AGG. One HA tag and an NLS were subsequently added by PCR using the following primers: 5'-CGA ATT AAC CCT CAC TAA AGG GAT TTC CAA GTC TCC ACC and 3'-TCA CAG CTT TCG CTT CTT CTT TGG CGC ATA GTC AGG AAC ATC.

In vitro transcriptions were performed as described in Beenders et al. (2007). Template DNAs were obtained by linearizing the clones described above downstream of their respective ORF, and either SP6 or T7 polymerases, as required, were used to synthesize capped, sense strand RNAs. Newly made RNAs were phenol-chloroform extracted, precipitated with ethanol, and resuspended in water. Their concentration was adjusted to $\sim 1 \mathrm{mg} / \mathrm{ml}$.

Oocytes and microinjection

Female adult frogs (Xenopus laevis) were anesthetized in $0.15 \%$ tricaine methanesulfonate (MS222,
Sigma Chemical, St. Louis, MO, USA), and small fragments of ovary were surgically removed. Oocytes were defolliculated by treating the ovarian fragments with $0.2 \%$ collagenase (type II, Sigma) in the saline buffer OR2 (Wallace et al. 1973), for $2 \mathrm{~h}$ at room temperature. Stage IV-V oocytes were selected under a dissecting microscope and maintained for up to 3 days in OR2 at $18^{\circ} \mathrm{C}$. The glass needles used for microinjection were prepared using a horizontal pipette puller P-97 (Sutter Instrument, Novato, CA, USA) and capillary glass tubes $(0.5 \mathrm{~mm}$ i.d. and $1.2 \mathrm{~mm}$ o.d.). All injections were performed under a dissecting microscope (S6 Leica) using a nanojet II (Drummond, Broomal, PA, USA). Cytoplasmic and nuclear injections had a constant volume of $30 \mathrm{nl}$ and $4.8 \mathrm{nl}$, respectively.

Sperm nuclei preparation and injection

Sperm heads of Xenopus laevis were kindly provided by Dr. Peter Jones (UIUC). They were prepared from testes as described in Newmeyer and Wilson (1991); and were demembranated with lysolecithin using the original procedure described in Gurdon et al. (1976). The injection needles were filled with 2-4 $\mu$ l of sperm suspension and were microinjected directly into the nuclei of defolliculated oocytes. We aimed at injecting between 5 and 10 sperm heads per oocyte. We found that an accurate number of sperm heads could only be injected within the first 10 minutes. After that critical period, as noted in Gall and Murphy (1998), we observed that the number of injected sperm heads decreased rapidly, presumably because of aggregation, adherence to the side of the needle glass, and gravity-driven deposition to the bottom side of the needle. The only preparation of the oocytes was to position them so that the center of the animal pole would correspond to the entry point of the needle. With a little practice, we obtained an 80 $90 \%$ success rate, which is quite adequate for our studies.

\section{Oil-isolation of nuclei and FRAP}

Non-defolliculated oocytes were injected with RNA coding for the YFP-hRAD21-3HA proteins, and the isolation of nuclei in mineral oil was performed $24 \mathrm{~h}$ later as described in Patel et al. (2008). In some cases, oocytes were also injected with DAPI (20 $\mathrm{nl}$ of a 
$5 \mathrm{ng} / \mu \mathrm{l}$ solution of DAPI in water) $5 \mathrm{~min}$ prior nuclei isolation to confirm the chromosomal distribution of YFP-hRAD21-3HA. FRAP was performed using the SRS NL100 MicroPoint Laser System (Photonic Instruments Inc., St. Charles, IL, USA) adapted to our DMR microscope. A $514 \mathrm{~nm}$ laser was used for the photobleaching of YFP. Data acquisition and quantitation was done using the In Vivo software (version 3.2.0, Media Cybernetics, Bethesda, MD, USA).

Nuclear spreads and immunofluorescence

Nuclear spreads were prepared as described in Gall and Murphy (1998). Preparations were fixed in phosphate-buffered saline (PBS) containing 2\% paraformaldehyde and $1 \mathrm{mM} \mathrm{MgCl} 2$ for $1 \mathrm{~h}$ at room temperature. After fixation, preparations were rinsed briefly in PBS and blocked for $10 \mathrm{~min}$ in PBS containing $0.5 \%$ BSA (Sigma) and $0.5 \%$ gelatin (from coldwater fish). Spreads were then incubated with primary and secondary antibodies diluted in blocking buffer for $1 \mathrm{~h}$, respectively, washed in $1 \times$ PBS and mounted in $50 \%$ glycerol containing $10 \mathrm{pg} / \mathrm{ml}$ of 4',6diamidino-2-phenylindole (DAPI) and $1 \mathrm{mg} / \mathrm{ml}$ of phenylenediamine (Sigma). The following Alexa Fluor ${ }^{\circledR}$ conjugated antibodies (Invitrogen) were used at a concentration of $2.5 \mu \mathrm{g} / \mathrm{ml}$ : Alexa 488 goat antirabbit IgG; Alexa 594 goat anti-mouse IgG; Alexa 594 goat anti-rat IgG; and Alexa 488 goat anti-mouse IgG. The anti-HA antibody, mAb 3F10 (Hoffmann-La Roche Inc., Nutley, NJ, USA), was used at $50 \mathrm{ng} / \mathrm{ml}$. The anti-Xenopus SMC3 antibody was kindly provided by Dr. T. Hirano (Cold Spring Harbor Laboratory) and used at a dilution of 1:100. The anti-XCAPD2 antibody (serum $\mathrm{G}$ ) was used as described in Beenders et al. (2003). The anti-SMC3 (A300-060A), SMC1, SMC2 and SMC4 antibodies were purchased from Bethyl Laboratories, Inc. (Montgomery, TX, USA). Coilin was detected using the monoclonal antibody mAb H1 (Tuma et al. 1993).

\section{Western blots}

Whole oocytes, cytoplasms, and nuclei were handisolated and homogenized in $83 \mathrm{mM} \mathrm{KCl}, 17 \mathrm{mM}$ $\mathrm{NaCl}, 6.5 \mathrm{mM} \mathrm{Na} \mathrm{HPO}_{4}, 3.5 \mathrm{mM} \mathrm{KH} \mathrm{PO}_{4}, 1 \mathrm{mM}$ $\mathrm{MgCl}_{2}, 1 \mathrm{mM}$ dithiothreitol, $\mathrm{pH}$ 7.0. Crude extracts were centrifuged at $22000 \mathrm{~g}$ at $4^{\circ} \mathrm{C}$ for $10 \mathrm{~min}$. Clarified extracts were collected and fractionated on
$5-15 \%$ polyacrylamide-SDS gradient gels, using the Mini-PROTEAN 3 Electrophoresis System (Bio-Rad, Hercules, CA, USA). Proteins were transferred to Immobilon membranes (Millipore, Bedford, MA, USA) for $2 \mathrm{~h}$ at $40 \mathrm{~V}$ in Tris-glycine buffer containing $20 \%$ methanol. Membranes were blocked in 5\% dry milk-PBS for $1 \mathrm{~h}$ at room temperature and then incubated for $1 \mathrm{~h}$ at room temperature in PBS-Tween (PBST) containing the primary antibody. Membranes were washed 3 times with $1 \times$ PBS containing $0.05 \%$ Tween 20 (PBS2T) and incubated with the secondary antibody in PBST for $1 \mathrm{~h}$ at room temperature. After 3 washes with PBS2T, detection was performed using the ECFTM kit (GE Healthcare, Fairfield, CT, USA) and the FLA3000 fluoroimager (Fuji Medical Systems, Stamford, CT, USA). Either anti-Xenopus SMC1-4 antibodies (provided by Dr. T. Hirano; Cold Spring Harbor Laboratory) or anti-SMC1 (BL308) and antiSMC3 (BL313) antibodies (Bethyl Laboratories, Inc.) were used to detect SMC subunits. The anti-RAD21 (BL331) antibody was purchased from Bethyl Laboratories, Inc. Secondary antibody (alkaline phosphatase conjugated goat anti-rabbit antibody) was purchased from Sigma Chemicals.

Microscopy and image acquisition

Microscopy was performed using an upright Leica DMR (Heidelberg, Germany) and the following two objectives: PL Fluotar $40 \times$ oil objective $(\mathrm{NA}=1.0)$ and a HCL FL Fluotar 100× oil objective (NA=1.30). Both transmitted light and fluorescent images were captured using a monochrome Retiga EXI ChargeCoupled Device (CCD) camera (Qimaging, Surrey, $\mathrm{BC}$, Canada) driven by the In Vivo software (version 3.2.0, Media Cybernetics). Fixed nuclear spreads as well as oil-isolated nuclei were imaged at $20^{\circ} \mathrm{C}$. All images were processed using Adobe Photoshop CS version 8.0 and assembled with Adobe InDesign CS version 3.0 .

\section{Results}

Newly expressed hRAD21 is recruited to LBCs where it co-localizes with endogenous SMC3

To investigate which of the known cohesins might be associated with LBCs, we tested numerous anti- 
cohesin subunit antibodies, but only two produced a significant chromosomal labeling. These antibodies (clone A300-060A and $\alpha-X l \mathrm{SMC} 3$ ) are purified rabbit polyclonal sera directed against the mammalian and amphibian SMC3 proteins, respectively. A strong signal was observed on the chromosomal axes, primarily associated with the chromomeres (see Fig. 2 for a typical example using $\alpha-X l \mathrm{SMC} 3$ ). These chromomeres are regions where sister chromatids are tightly paired. Thus, the $\alpha-X l \mathrm{SMC} 3$ staining pattern is
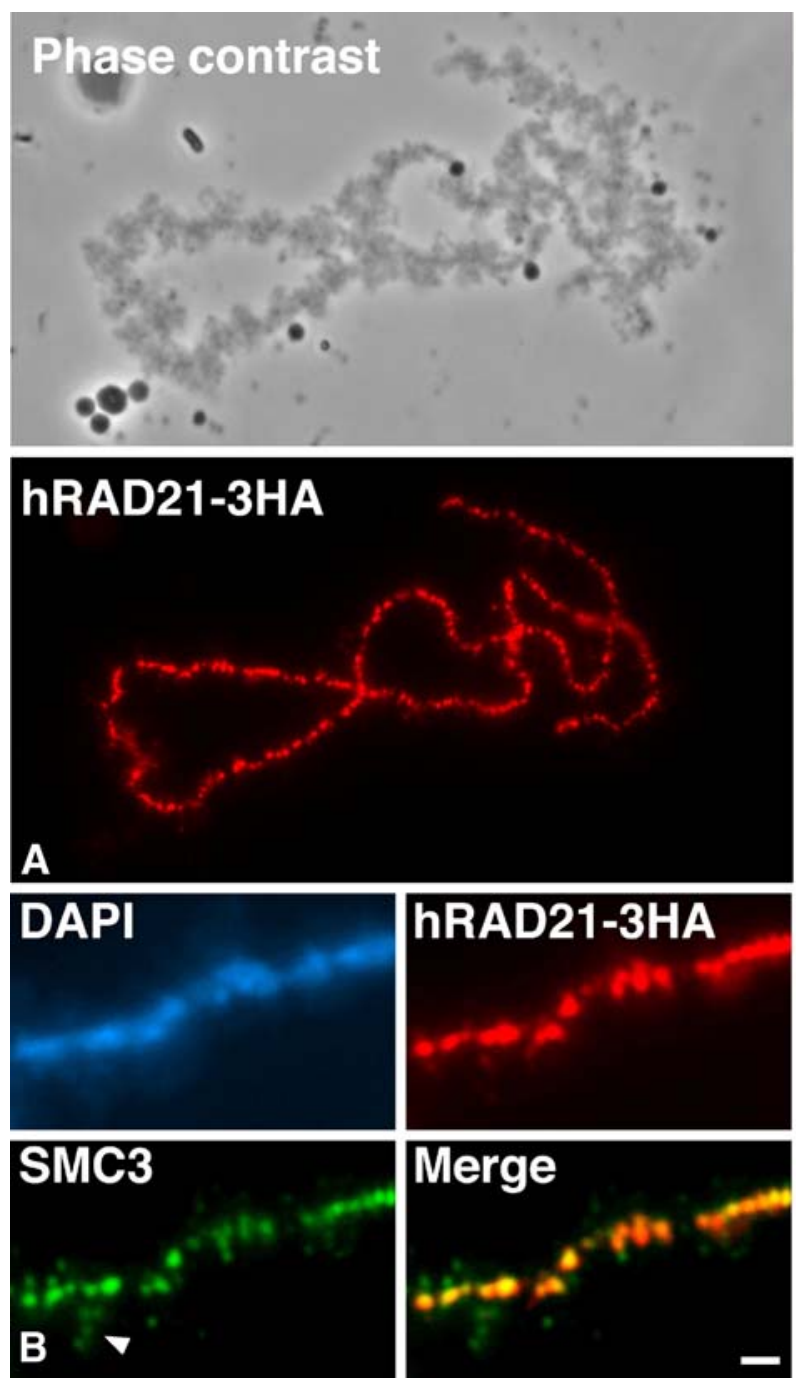

Fig. 2 Chromosomal distribution of SMC3 and hRAD21. (A) Transcripts coding for hRAD21-3HA were injected into stage IV oocytes and nuclear spreads were prepared $18 \mathrm{~h}$ later. The newly made hRAD21-3HA (red) and endogenous SMC3 (green) were detected using mAb 3F10 and the anti-XISMC3 antibodies, respectively. Both proteins display extensive co-localization on the chromosomal axes. DNA was counter- that expected for cohesin on LBC chromatids. In addition, a much weaker labeling was also occasionally observed on the lateral loops. However, it did not correspond to the chromatin component, but rather appears as small dots associated with the RNP matrix (arrowhead in Fig. 2B). While SMCs are multifunctional proteins, they are not involved in RNA processing. Importantly, an identical staining pattern of the chromosomal axes was obtained with the A300-060A antibody (data not shown), which indi-
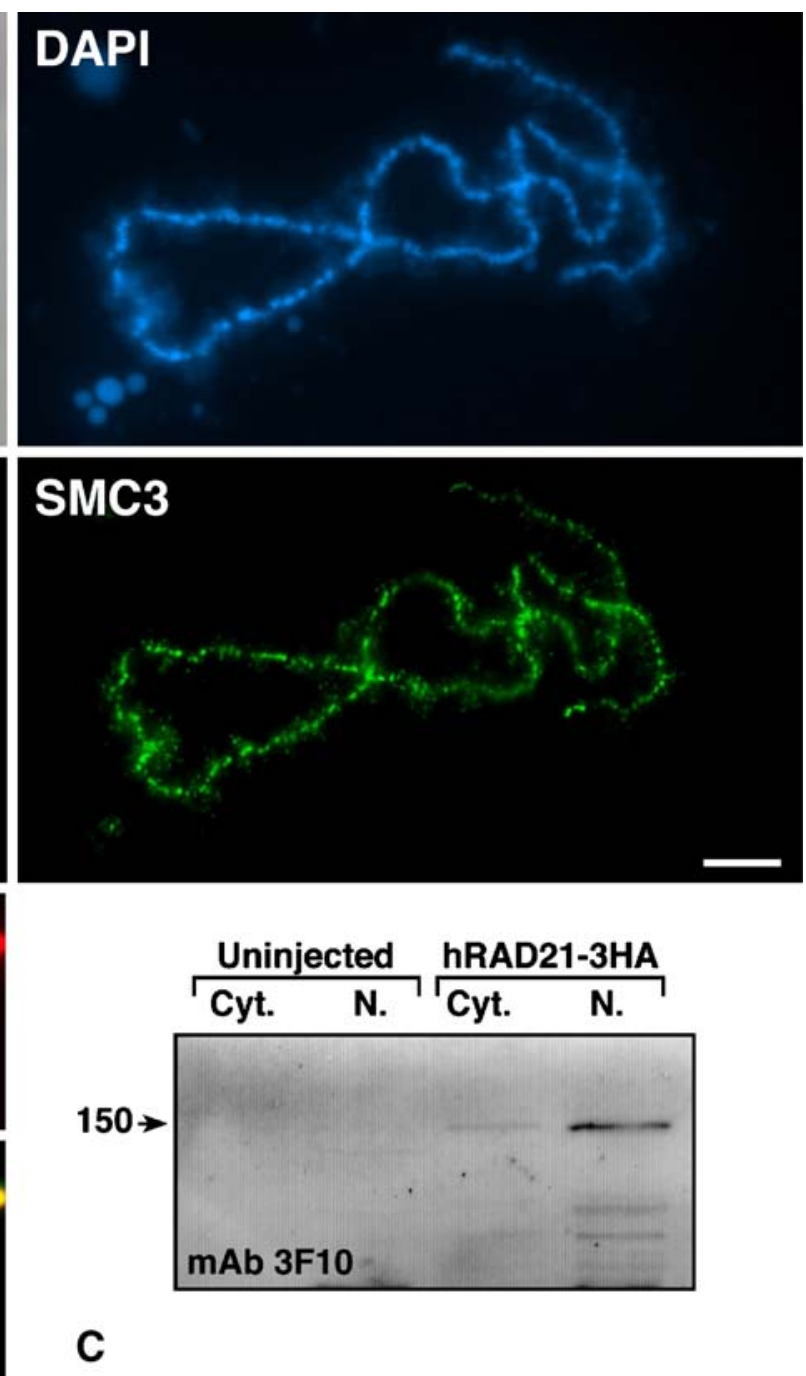

stained with DAPI (blue). Scale bar represents $10 \mu \mathrm{m}$. (B) A magnified view of the axis of one of the two homologues seen in (A) shows that the association of hRAD21-3HA and SMC3 with LBCs is essentially restricted to chromomeres. Scale bar represents $1 \mu \mathrm{m}$. (C) Western blot showing that the newly expressed hRAD21-3HA, detected using mAb 3F10, has an apparent molecular weight of $150 \mathrm{kDa}$ and is primarily nuclear 
cates that the epitope detected in the chromomeres corresponds to the endogenous SMC3, rather than an artifact. Cohesin has been shown to play a role in transcriptional regulation by virtue of its proposed role in looping chromatin to modulate enhancer and promoter proximity (Dorsett 2007). It may be that this punctate staining reflects a cohesin population engaged in transcriptional regulation at a number of loci.

We next wanted to examine the localization of other cohesin subunits to confirm that our observed axial SMC3 localization was indeed indicative of cohesin binding. In the absence of the appropriate antibodies to study other cohesin subunits on LBCs, we expressed several members of cohesin tagged with the hemagglutinin (HA) epitope. We first present the results obtained with the human RAD21 fused to three HA tags at its carboxyl end. The functional conservation of RAD21 among vertebrates makes it likely that the human version of RAD21 functions in Xenopus oocytes. Capped, sense-strand RNAs coding for hRAD21-3HA were synthesized in vitro and injected into the cytoplasm of stage IV-V oocytes. Nuclei were isolated at various time points after injection for nuclear spread preparations and western blot analysis. In both cases, the newly made hRAD21-3HA protein was detected using the monoclonal antibody mAb3F10, which displays very high specificity and affinity for the HA epitope and yields essentially no detectable fluorescent signal in control oocytes (data not shown; Gall et al. 1999; Beenders et al. 2007; Patel et al. 2007). hRAD21-3HA protein could be detected in the nucleus as a protein of $\sim 150 \mathrm{kDa}$ and localized to LBCs as early as $1 \mathrm{~h}$ after injection. Thus, hRAD21-3HA translation, nuclear import, and chromosome targeting occurred very efficiently. hRAD21-3HA continued accumulating in the nucleus, but even after $24 \mathrm{~h}$ of incubation LBCs remained the only labeled structures (Fig. 2). On LBCs, hRAD21-3HA associated specifically with the chromomeres, where it displayed an extensive co-localization with SMC3. In addition, the strength of both SMC3 and hRAD21-3HA signals was directly correlated to the relative density of the DNA present in each chromomere as defined by DAPI staining. Importantly, the chromosomal distribution of SMC3 in hRAD213HA expressing oocytes and uninjected control oocytes is identical (data not shown). Thus, two cohesin subunits are observed within the same regions of LBCs where sister chromatids cohesion exists.
Taken together, these data indicate that high levels of cohesin localize at chromomeres along the entire axial length of LBCs.

Since chromomeres correspond to regions of condensed chromatin, the chromosomal distribution of hRAD21-3HA was also compared to the pattern of XCAP-D2, a subunit of the condensin complex, which is involved in chromosome condensation (Hirano et al. 1997). We found that these two factors precisely co-localized within chromomeres (Fig. 3A). In addition, we followed the fate of the two proteins during chromosome condensation. Oocytes were treated with actinomycin D (AMD) for several hours to inhibit both RNAPII and RNAPIII transcriptional activities. Treatment of oocytes with AMD, or other transcriptional inhibitors, results in a dramatic retraction of the lateral loops and an overall condensation of the LBCs. This effect is reminiscent of the fact that in oocytes approaching the stage of maturation, transcription stops and LBCs condense to prepare for the first meiotic metaphase. We observed that hRAD21-3HA and XCAP-D2 remained strictly co-localized in LBCs during the various stages of chromatin condensation (as exemplified in Fig. 3B).

Is the recruitment of newly made hRAD21-3HA to $\mathrm{LBCs}$ dependent on chromosomal SMC1 and SMC3?

To investigate how hRAD21 binds to chromosomes, we first carried out a deletion analysis of hRAD21 to identify a possible chromosome-targeting domain. Studies in budding yeast have shown that the MCD1/ RAD21 N-terminus binds to the SMC3 head domain, whereas its C-terminus binds to the SMC1 head domain (Haering et al. 2002; Gruber et al. 2003). We therefore generated a series of hRAD21 clones bearing $\mathrm{N}$-terminal or $\mathrm{C}$-terminal deletions to assess the importance of the SMC interaction domains (ID) on LBC targeting. Both N-terminal and C-terminal truncated forms of hRAD21 were expressed in stage IV-V oocytes, and their association with LBC chromomeres was monitored by fluorescence microscopy. The results are summarized in Fig. 4, and several conclusions can be drawn from them. First, the recruitment of hRAD21 to LBCs is greatly influenced by its SMC1 and SMC3-interacting domains (ID), as well as by a central region containing at least residues 288-467, which may overlap with the SCC3 inter- 


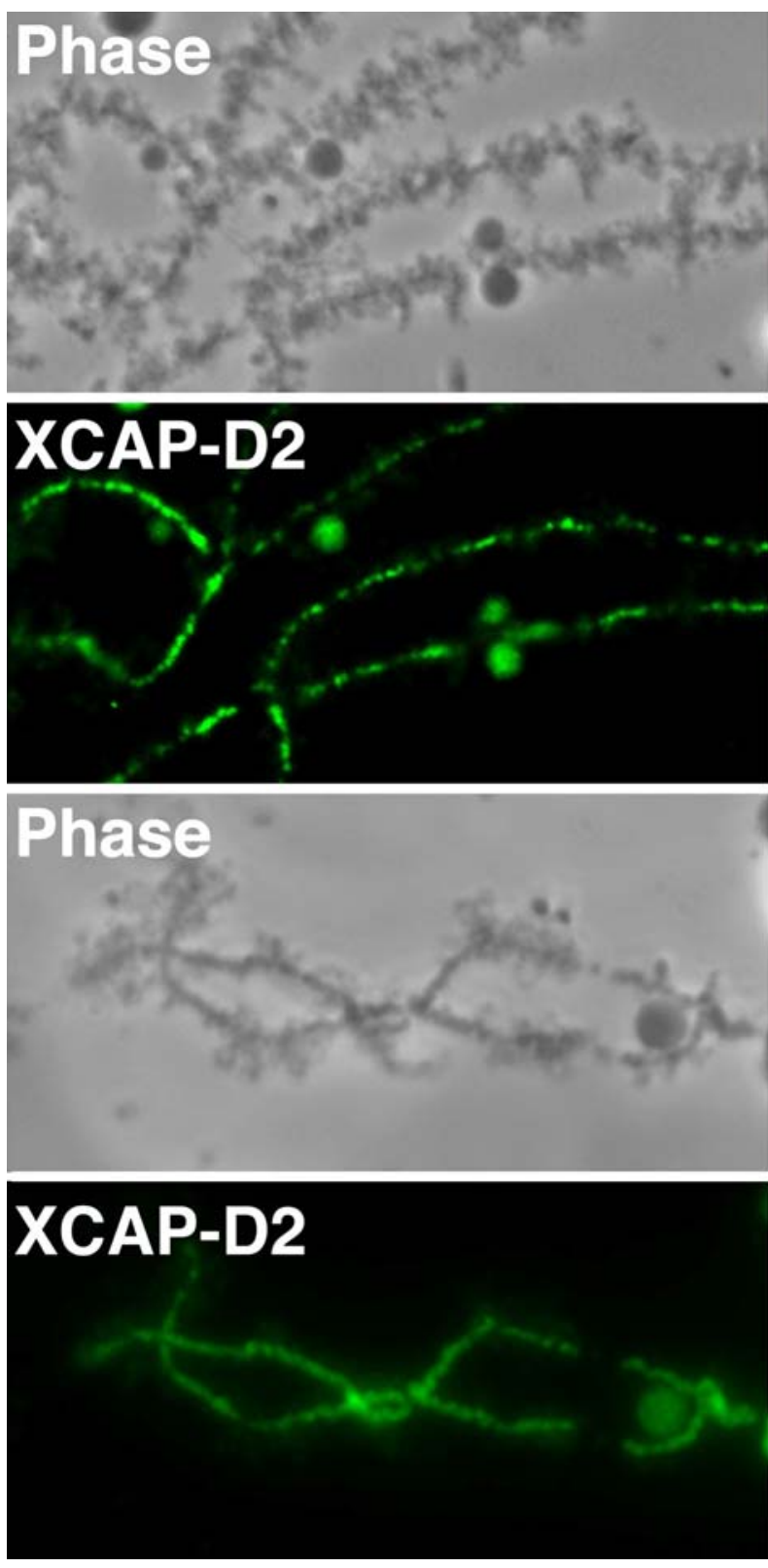

Fig. 3 hRAD21 co-localizes with XCAP-D2 on LBCs. (A) Transcripts coding for hRAD21-3HA were injected into stage IV oocytes and nuclear spreads were prepared $18 \mathrm{~h}$ later. The newly made hRAD21-3HA (red) and endogenous XCAP-D2 (green) were detected using $\mathrm{mAb} 3 \mathrm{~F} 10$ and an antibody directed against the Xenopus CAP-D2, respectively. Both proteins were co-localized on the LBC axes. Scale bar

acting region identified in yeast (Gruber et al. 2003). Second, while the deletion of the SMC1-ID completely prevented targeting to LBCs, the removal of the SMC3-ID only weakened it. Importantly, hRAD21 does not exhibit a discrete domain that is
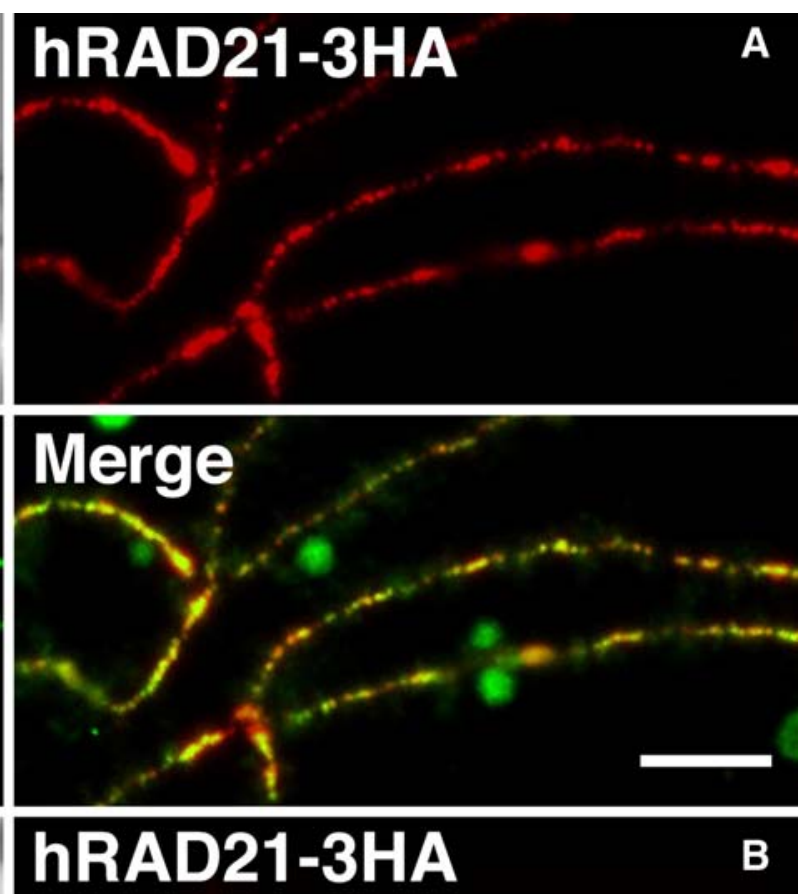

\section{Merge}

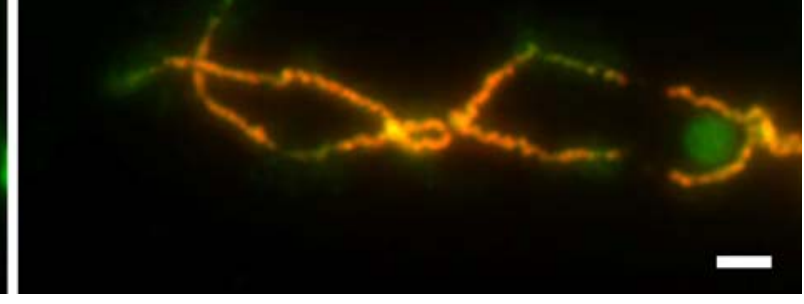

represents $5 \mu \mathrm{m}$. (B) Some of the oocytes used above were AMD treated for $2 \mathrm{~h}$ before preparing nuclear spreads. Many of the loops of the LBC shown here have already retracted. In addition, the chromosomal axes are considerably shortened. During these structural rearrangements both hRAD21 and XCAP-D2 remain specifically associated with the axes. Scale bar represents $5 \mu \mathrm{m}$

necessary and sufficient to interact with LBCs. Taken together, these data suggest that the overall conformation of hRAD21 and/or multiple interactions with the other cohesin members are required to load hRAD21 onto LBCs. 

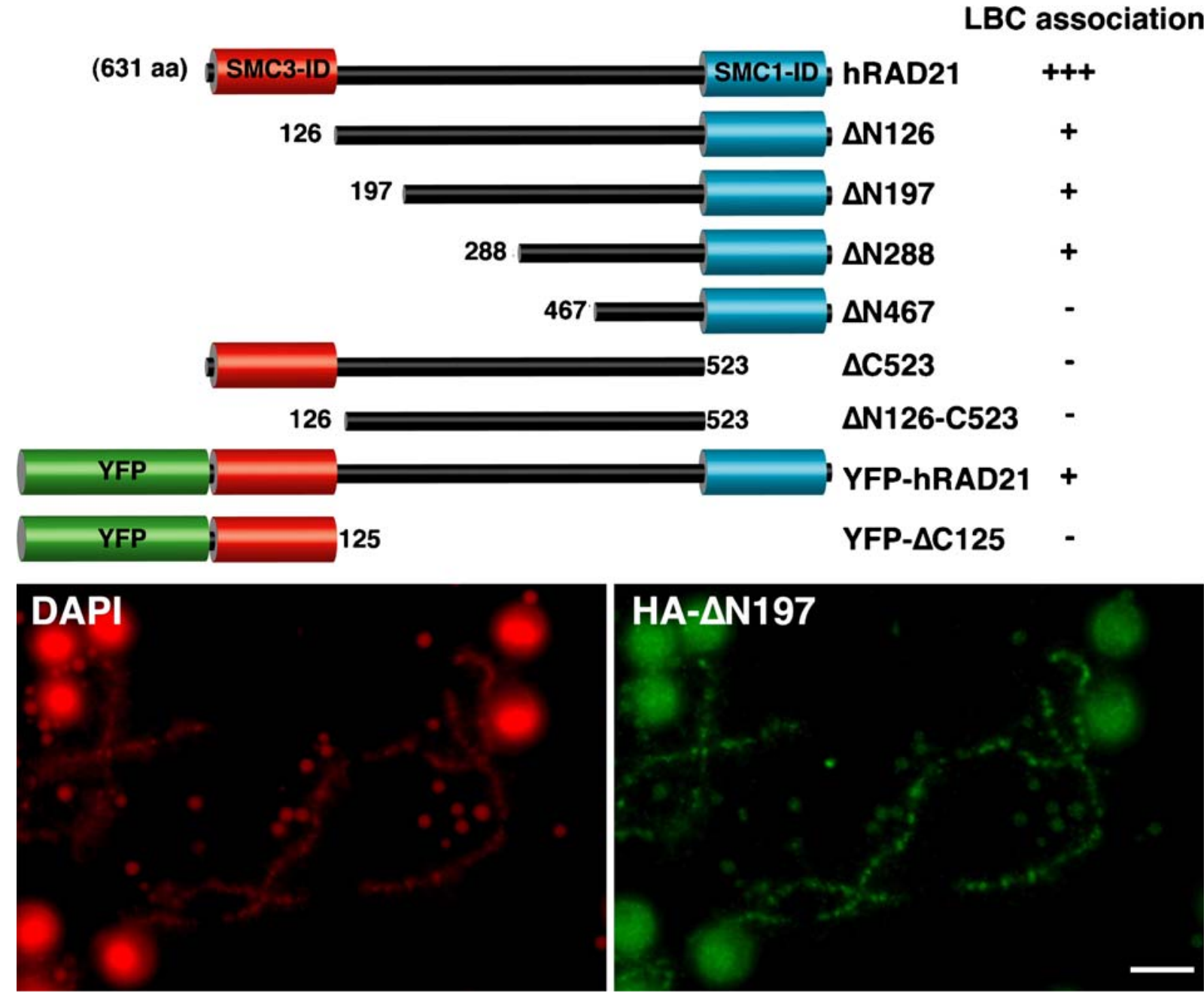

Fig. 4 Deletion analysis of hRAD21 reveals several critical regions for its chromosomal recruitment. The various truncated hRAD21 mutants generated for this study are presented schematically. Numbers refer to amino acids and indicate the extent of the truncations. These mutants were tagged with three HA epitopes and expressed in stage V oocytes. Their chromosomal distribution was then analyzed on fixed nuclear

These results prompted us to investigate the cellular distribution of SMC1 and SMC3 in the oocyte. Nuclei were separated from the cytoplasm of stage $\mathrm{V}$ oocytes, and the respective soluble nucleoplasmic and cytoplasmic protein fractions were tested by western blot for the presence of both SMCs. Surprisingly, SMC1 and SMC3 were readily detected in the cytoplasm but were absent from the nucleoplasm (Fig. 5). In contrast, the condensin subunits SMC2 and SMC4 were present in both soluble fractions. The protein coilin, which is primarily

spreads by indirect immunfluorescence, using the antibody $\mathrm{mAb} 3 \mathrm{~F} 10$. Results are indicated relative to the chromosomal association of full length hRAD21-3HA. The distribution of mutant $\triangle 197-3 H A$ is presented as a typical example of a weak $(+)$ chromosomal interaction. Note that nucleoli are also brightly labeled, most likely in a non-specific way. Scale bar represents $10 \mu \mathrm{m}$

nuclear and an abundant resident of the nucleoplasm (Bellini 2000), was used as a positive control.

This result suggested at least two possibilities, which are not mutually exclusive, regarding the association of hRAD21-3HA with LBCs. The first possibility is that newly made hRAD21-3HA associates with SMCs in the cytoplasm and enters the nucleus as a complex that is targeted to LBCs. The second possibility is that newly made hRAD21-3HA enters the nucleus independently from the cytoplasmic pool of cohesin subunits. Upon nuclear entry, 


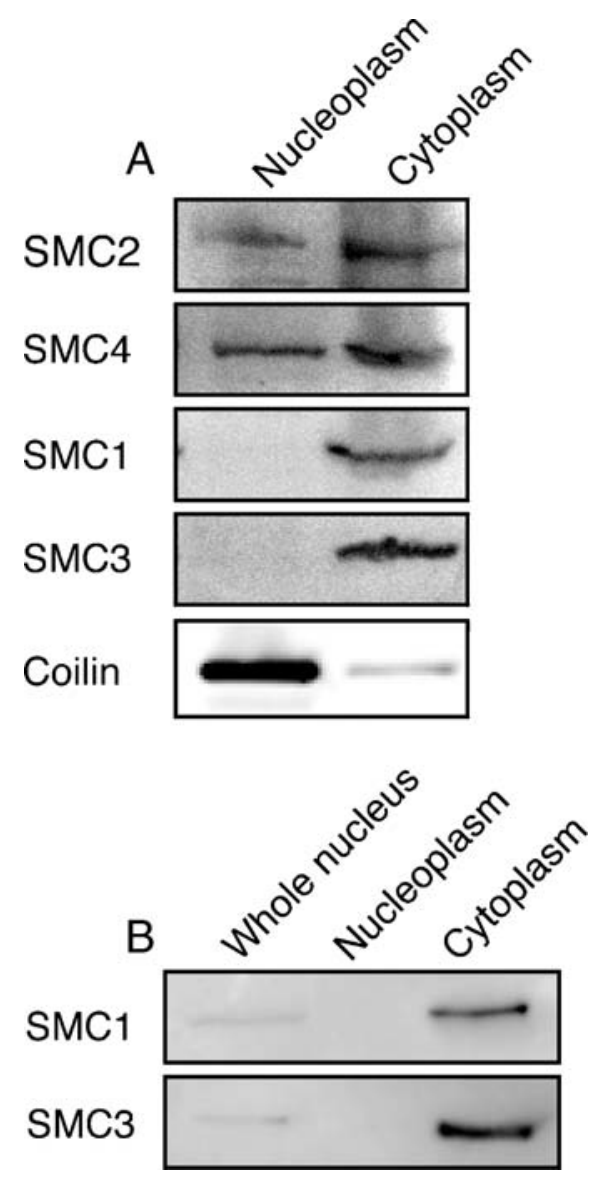

Fig. $5 \mathrm{SMC} 1$ and SMC3 are not detected in the nucleoplasm. (A) Soluble nucleoplasmic and cytoplasmic proteins were prepared from 20 oocytes and analyzed by western blotting using antibodies directed against various SMCs. While SMC2 and SMC4 are detected in both protein fractions, SMC1 and SMC3 could only be seen in the cytoplasmic fraction. Coilin is used here as a representative protein that is abundant in the nucleoplasm, and was detected using the monoclonal antibody $\mathrm{mAb} \mathrm{H} 1$. (B) Nucleoplasmic and whole-nuclear proteins were prepared from 50 oocytes. SMC1 and SMC3 were detected as in (A). The cytoplasmic lane corresponds to 20 oocytes. The signals observed in the whole nuclear protein extract correspond to chromosomal associated SMC1 and SMC3

hRAD21-3HA binds LBCs through interactions with chromosomal SMC1 and SMC3. To distinguish between these two hypotheses, we took advantage of the fact that the nucleus of an oocyte can be readily isolated from the cytoplasm under mineral oil. The hydrophobic environment of the oil prevents any loss of nucleoplasm while allowing for gas exchange. As a result, an oil-isolated nucleus maintains all its structures and functions for several hours and longer if provided with a source of ATP (Lund and Paine 1990;
Paine et al. 1992). We produced hRAD21-3HA protein in vitro and directly injected it into oil-isolated nuclei. After $1 \mathrm{~h}$ of incubation, injected nuclei were recovered into a saline buffer, and nuclear spreads were prepared. As a positive control, in vitro made hRAD21-3HA was also injected into the cytoplasm of stage $\mathrm{V}$ oocytes, and nuclear spreads were prepared $1 \mathrm{~h}$ later. In both cases, hRAD21-3HA was found associated with the LBC axes (Fig. 6). These results demonstrate that the cytoplasmic pool of SMC1 and SMC3 is not required for chromosomal loading of hRAD21-3HA. Instead, they suggest that newly made hRAD21-3HA is recruited to LBCs by SMC1 and SMC3 present in the nucleus. This could occur via RAD21 subunit exchange within cohesin already bound to chromosomes. Alternatively, cohesin may diffuse off chromosomes into the nucleoplasm, where RAD21 subunit exchange would occur prior to cohesin rebinding chromosomes.

Cohesins associate with newly formed, single, and unreplicated lampbrush chromatids

We next assayed whether cohesin subunits can associate with unreplicated chromatids. We utilized a powerful cytological assay, in which sperm heads are induced to form individual 'lampbrush chromatids' (Gall and Murphy 1998). In this experimental system, demembranated sperm heads are injected directly into the nucleus of oocytes. There, the highly compacted sperm chromatin initially swells and within a few hours recruits all the factors required to form 18 individual chromatids. Remarkably, these chromosomes display the same overall structure as the endogenous bivalent LBC, with an extended axis corresponding to a linear array of compacted chromomeres as well as numerous lateral loops transcribed by RNAPII (Gall and Murphy 1998). Importantly, since DNA replication is prevented in oocytes, these newly formed lampbrush chromatids have smaller chromomeres than the endogenous LBCs, and loops occur singly rather than in pairs.

With this chromatid formation assay, we found that both SMC3 and hRAD21-3HA associate specifically with newly formed lampbrush chromatids, where they extensively co-localize within chromomeres (Fig. 7). As we found using endogenous LBCs, this chromosomal pattern is unchanged for SMC3 in oocytes that do not express hRAD21-3HA. Since SMC3 is 


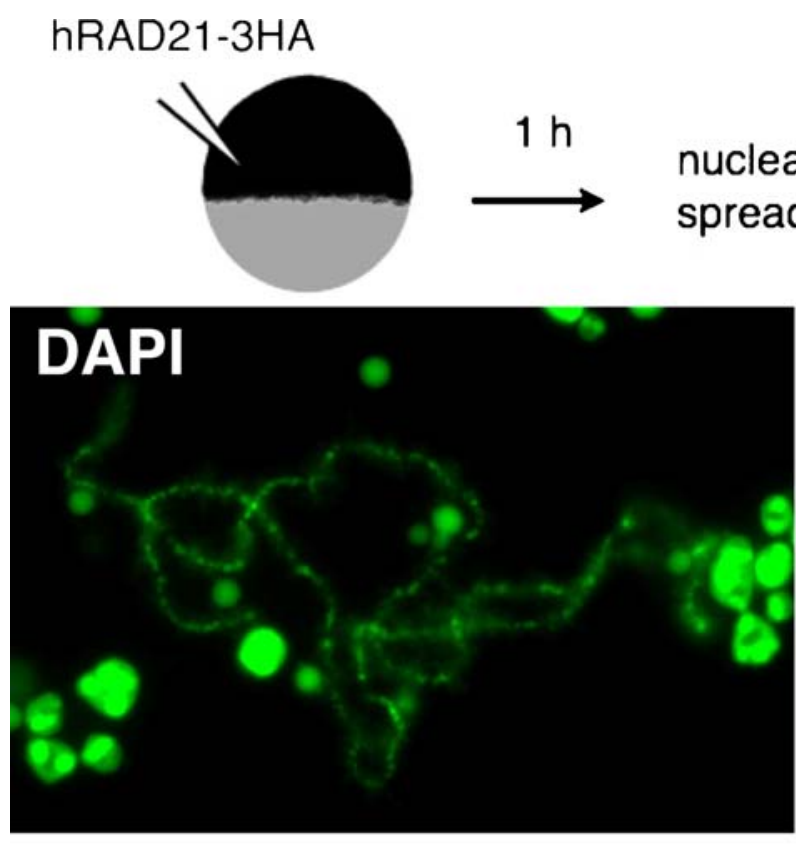

\section{hRAD21-3HA}

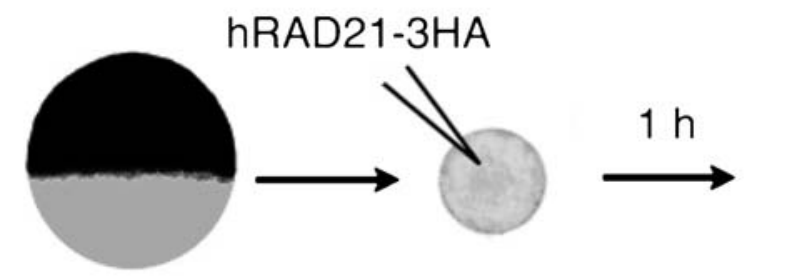

\section{nuclear \\ spread}

\section{DAPI}

\section{hRAD21-3HA}

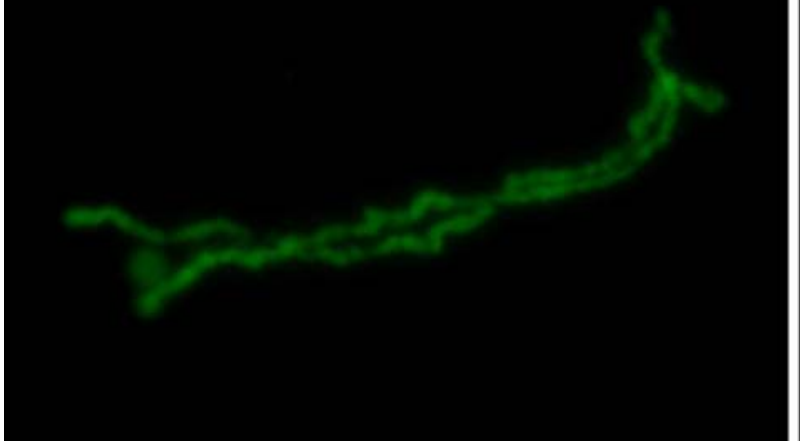

Fig. 6 hRAD21-3HA associates with LBCs in the absence of nucleoplasmic SMCs. In vitro made hRAD21-3HA protein was injected into stage V oocytes (top) or oil-isolated nuclei (bottom), as indicated schematically. Nuclear spreads were prepared 1

apparently absent from the nucleoplasm (Fig. 5) and the labeling of the endogenous LBCs is not significantly changed (Fig. 7), the injection of sperm heads must have resulted in a dynamic recruitment of the cytoplasmic SMC3, and presumably the complete

cohesin complex, to the nucleus for it to participate in the overall formation of lampbrush chromatids. Moreover, when several hundred sperm heads are injected into one nucleus, several thousand lampbrush chromatids form, and $\mathrm{SMC} 3$ is found associated

$\mathrm{h}$ later and the distribution of hRAD21-3HA was analyzed by indirect immunofluorescence, using $\mathrm{mAb} 3 \mathrm{~F} 10$. In both cases hRAD21-3HA targeted LBC chromomeres efficiently. Scale bars represent $10 \mu \mathrm{m}$ 

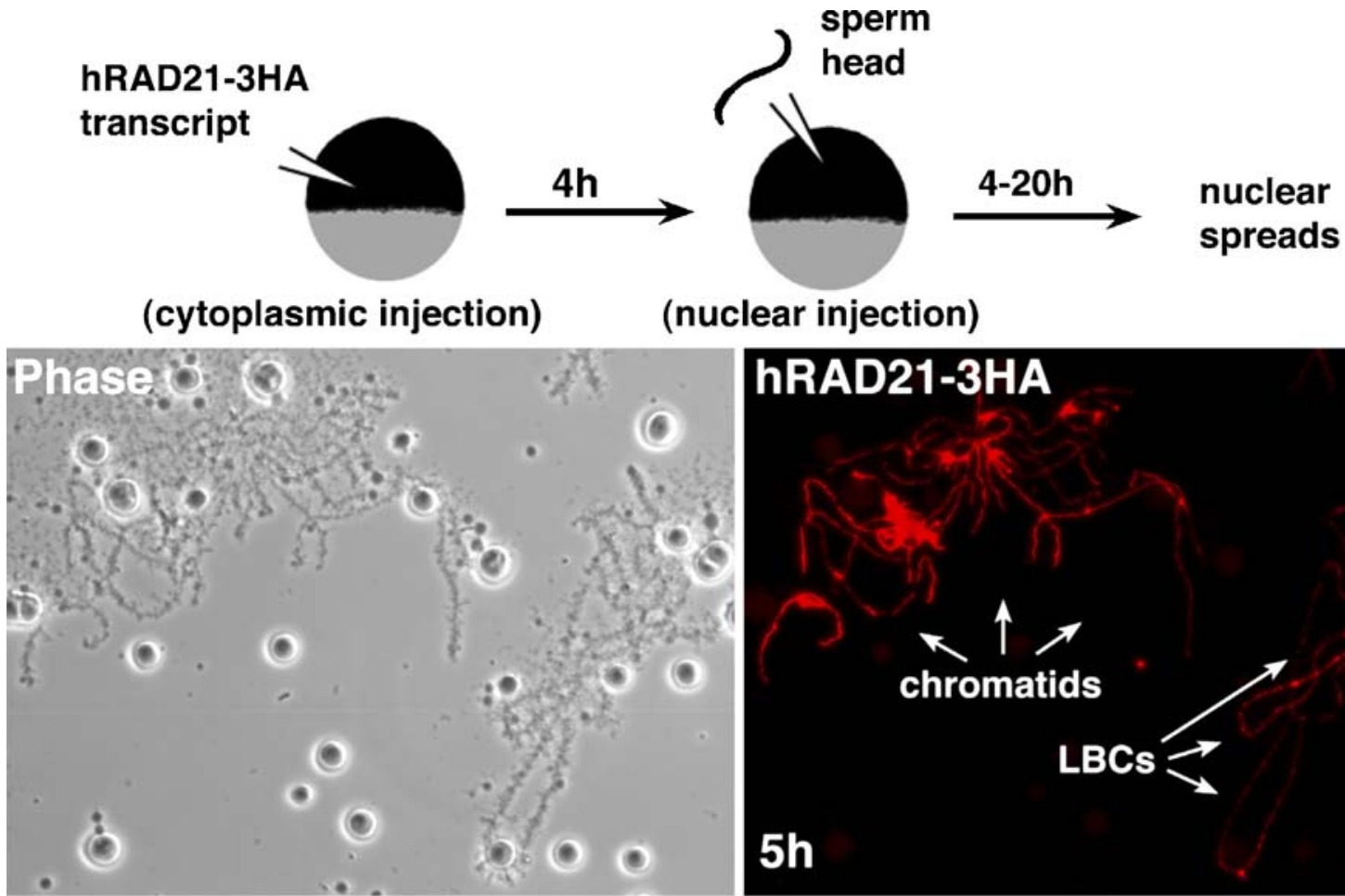

$5 h$

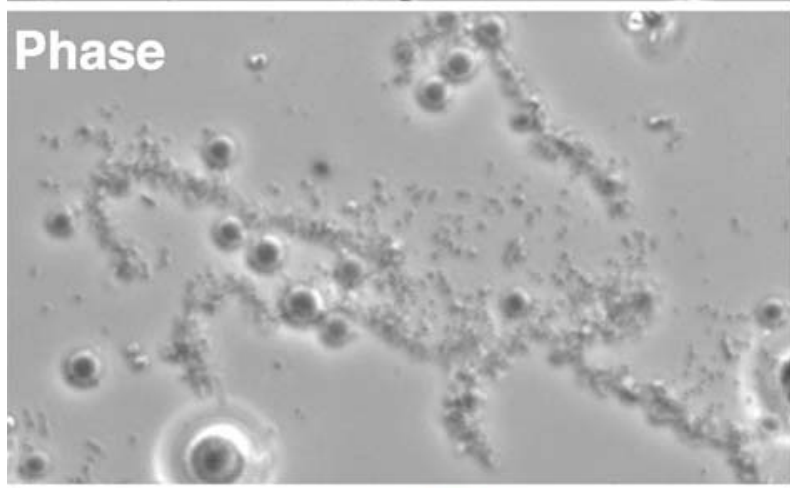

hRAD21-3HA

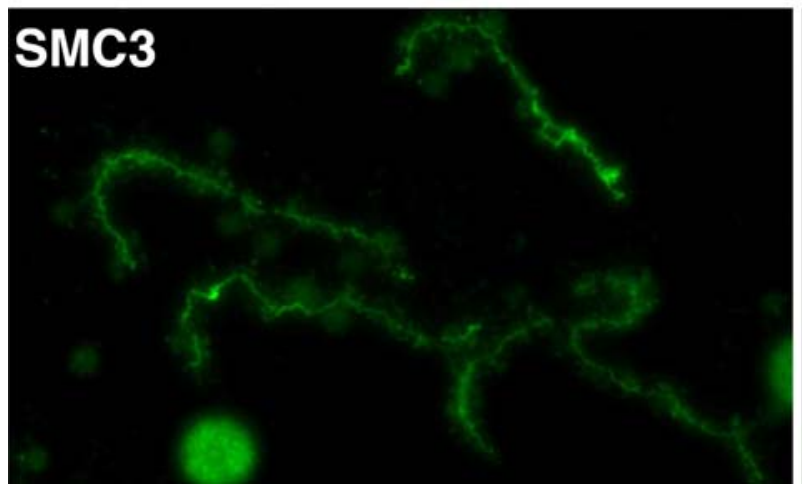

$20 \mathrm{~h}$

Fig. 7 Unreplicated, single lampbrush chromatids recruit cohesin subunits. Stage Voocytes were injected successively with hRAD213HA transcripts and demembranated sperm heads as indicated schematically. (A) Initially sperm heads swell and develop into clusters of threads (chromatids) that closely resemble LBCs. These

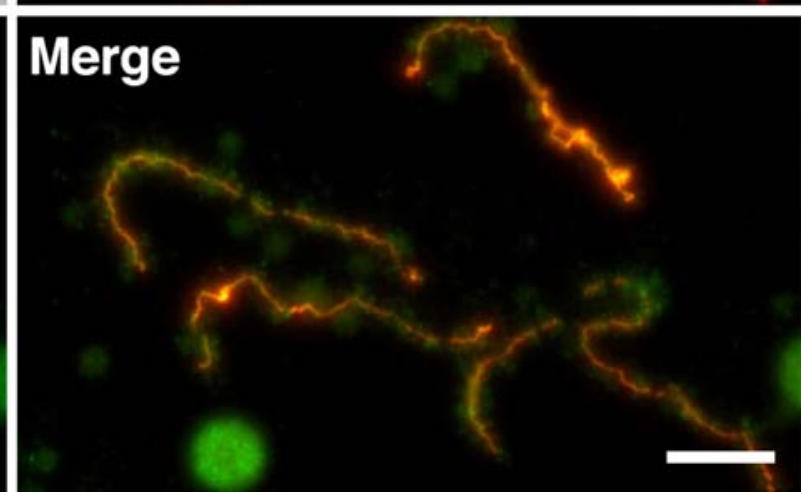

developing lampbrush chromatids recruit hRAD21-3HA specifically to their axes. (B) Eventually, these clusters resolve into individually recognizable lampbrush chromatids. Four chromatids are shown in the field and both hRAD21-3HA and endogenous SMC3 are co-localized on their axes. Scale bar represents $10 \mu \mathrm{m}$ 
within their chromomeres. The fact that SMC3 staining is robust on this large excess of lampbrush chromatids strongly argues against the idea that either a small nuclear pool of unbound cohesin or cohesin diffusing off the endogenous LBCs is responsible for the new single LBC chromatid staining. These data suggest that the recruitment of cohesin to the newly formed single chromatids is a precisely regulated event. It is also consistent with the idea that cohesin plays a role in the overall LBC architecture formation and or maintenance independently from its role in sister chromatid cohesion.

Dynamic exchanges of newly expressed hRAD21 between chromomeres and nucleoplasm

While nuclear spreads offer a high spatial resolution to visualize chromosomes, their standard preparation results in a complete loss of the nucleoplasm and prevents the study of LBC-associated protein steadystate dynamics. We recently optimized the procedure for imaging LBCs in oil-isolated nuclei by light microscopy (Patel et al. 2008), and we used this technique here to begin analyzing the dynamic properties of a newly expressed hRAD21-3HA fused to the yellow fluorescent (YFP) protein. Capped, sense strand transcripts coding for the YFP-hRAD213HA protein were injected into stage IV-V oocytes. After $18 \mathrm{~h}$ of incubation, nuclei were isolated in a saline buffer for nuclear spread preparations or under mineral oil for live fluorescence microscopy. On standard nuclear spreads, YFP-hRAD21-3HA associates specifically with the chromomeric regions of LBCs (Fig. 8B), in a pattern identical to that identified for hRAD21-3HA. Thus, the fusion of YFP to the amino-terminal end of hRAD21 does not interfere with its chromosomal binding. In oil-isolated nuclei, YFP-hRAD21-3HA was distributed between two domains, the nucleoplasm and the LBC (Fig. 8A). The fluorescent signal associated with a small axial region of a chromosomal axis was photobleached using a $514 \mathrm{~nm}$ laser beam (Fig. 8D). The same region was then imaged every $10 \mathrm{~s}$ for several minutes to monitor an eventual fluorescence recovery. Other chromosomal axes present in the field served as unbleached controls to take into account the loss of fluorescence of the overall sample inherent to the many image acquisitions. We found that the bleached chromomeres recovered a detectable fluorescent sig- nal within $90 \mathrm{~s}$, and it took about $8 \mathrm{~min}$ to reach $\sim 90 \%$ of the initial signal (Fig. $8 \mathrm{C}$ ). We attributed the rapid recovery of fluorescence to exchanges of bleached chromomeric YFP-hRAD21-3HA with fluorescent and nucleoplasmic YFP-hRAD21-3HA, rather than a lateral recruitment from the immediately adjacent chromomeres. We base this reasoning on the observation that the fluorescence of the flanking chromomeres was apparently not affected by the fluorescence recovery of the bleach area, and that there is a substantial pool of nucleoplasmic YFPhRAD21-3HA. Together, these data highlight the dynamic nature of the interactions between a newly expressed hRAD21 and LBCs.

\section{Discussion}

Little is known about the chromatin organization supporting the unique architecture of LBCs. In particular, many questions remain about the structure of chromomeres and the mechanisms regulating the formation and maintenance of the lateral loops that emerge from them. For instance, are chromomeres structured in looped chromatin domains as proposed by Joseph Gall in 1956? How are the sites of the transcriptionally active loop formation defined, and how is the absence of sister chromatin cohesion regulated there? Collectively, these questions can be seen as the major challenge to overcome in order to understand LBC structure and function. Clearly, a better definition of the LBC chromatin is needed to address these fundamental questions. Of high interest are proteins such as the SMCs, since they have emerged over the last decade as multifunctional proteins involved in many aspects of chromatin organization and dynamics, in particular chromatin condensation and cohesion (reviewed in Onn et al. 2008). In this study, we present original data on two members of the cohesin complex, RAD21 and SMC3. We show that both factors associate with LBCs, supporting the idea that cohesin participates in the overall dynamic organization of LBCs.

LBCs and the cohesin

We demonstrate that both SMC3 and hRAD21 associate with the LBC chromomeres. Interestingly, both SMC3 and hRAD21 are not detected in the 


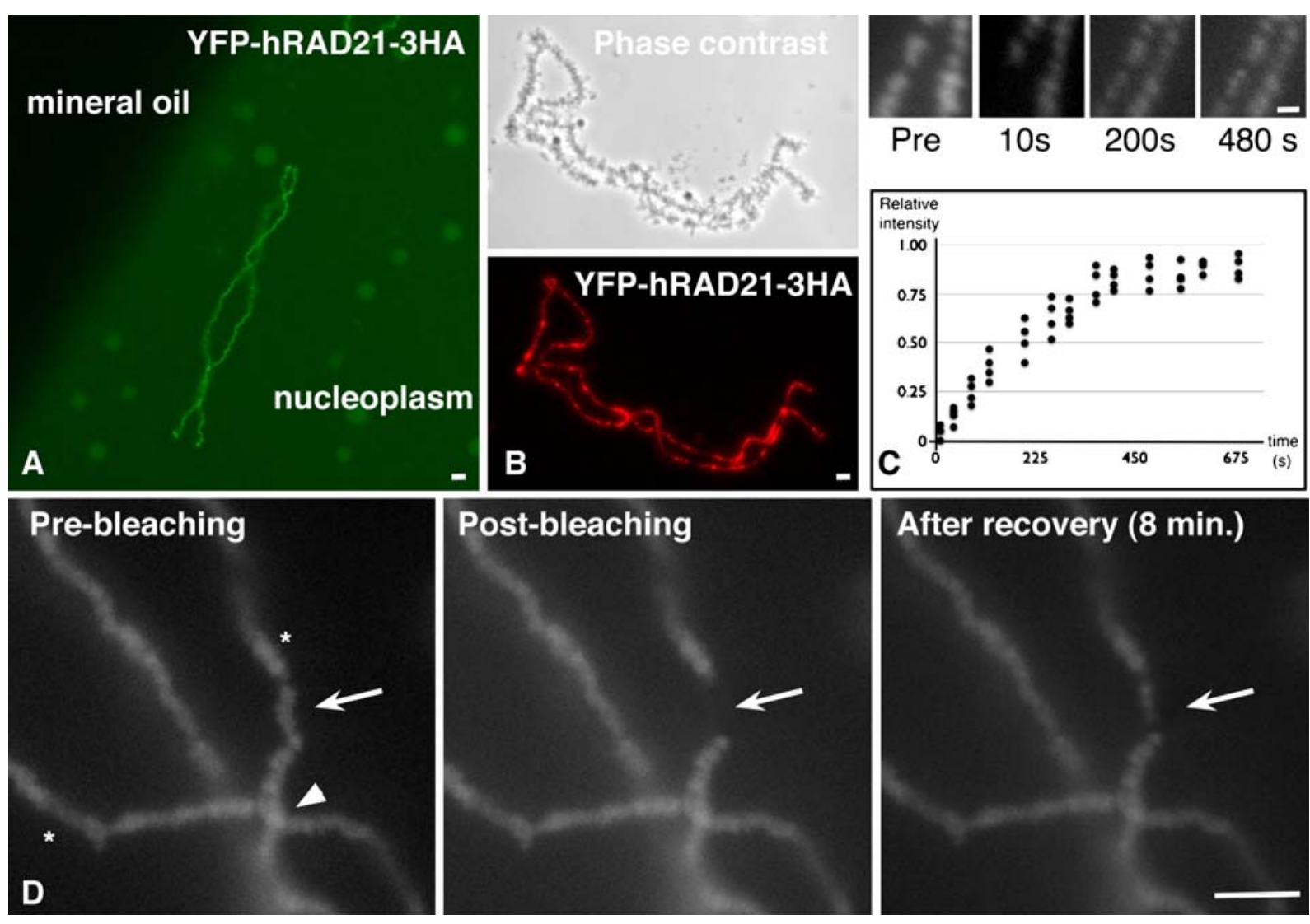

Fig. 8 Dynamic exchange of YFP-hRAD21-3HA between LBCs and nucleoplasm in oil-isolated nuclei. (A) Fluorescence micrograph showing the distribution of newly expressed YFP-hRAD21-3HA in a oil-isolated nucleus. As expected, YFP-hRAD21-3HA associates with chromosomal axes, but importantly is also present in the nucleoplasm. Scale bar represents $10 \mu \mathrm{m}$. (B) Phase-contrast micrograph and corresponding fluorescent image showing the chromosomal distribution of YFP-hRAD21-3HA on fixed LBCs. Scale bar represents $10 \mu \mathrm{m}$. (C) Images showing a narrow region of an LBC where chromomere-associated YFP-hRAD21-3HA was

chromatin regions interspaced with the chromomeres. Sister chromatids are also paired in these connective chromatin regions (Callan 1986). Thus, one interpretation is that the level of DNA compaction is too low to promote a signal that can be detected by fluorescence microscopy. It is unlikely that a lack of staining of the interchromomeric region is due to epitope masking, since two different SMC3 antibodies and hRAD21-3HA failed to detectably stain these regions. In the case of RAD21, we base our present study on its expression as HA- or YFPhRAD21 fusion proteins. Thus, in the absence of probes that specifically detect the endogenous photobleached. Fluorescence recovery was then followed over time. Scale bar represents $2 \mu \mathrm{m}$. A FRAP curve of YFPhRAD21-3HA corresponding to four distinct chromomeres is presented below. In each case, the intensity of the bleach spot was measured as a function of time. (D) Fluorescence bleaching and recovery of chromosomal YFP-hRAD21-3HA. A narrow region (arrows) of one of the two homologues (asterisks) forming an LBC was photobleached using a $514 \mathrm{~nm}$ laser beam and recovery of fluorescence was monitored over time. Scale bar represents $10 \mu \mathrm{m}$

RAD21 in situ, we cannot exclude the possibility that RAD21 has in fact a different chromosomal distribution from the one defined here for hRAD21-3HA. This is unlikely to be the case, however, since our results suggest that the association of hRAD21 with LBCs depends in part on its ability to interact with chromosomal SMC1 and SMC3. Finally, the same labeling restriction to axial chromomeres was previously observed for XCAP-D2, a member of the condensin I complex, using three different antiXCAP-D2 antibodies (Beenders et al. 2003).

The fact that we find endogenous SMC3, a subunit of both mitotic and meiotic cohesin complexes, to be 
present in chromomeres strongly suggests a role in the cohesion of the LBC sister chromatids. Our observations that epitope-tagged hRAD21 exhibits the same binding pattern also support this view. One critical aspect of cohesin biology is that cohesins must be loaded onto chromosomes prior to DNA replication to be functionally involved in sister chromatid cohesion (reviewed in Guacci 2007; Onn et al. 2008). However, cohesin can still be loaded onto chromosomes during mitosis, but these de novo loaded complexes are usually not capable of mediating cohesion (Haering et al. 2004). Therefore, despite our finding that newly hRAD21-3HA rapidly and specifically accumulates within chromomeres, it is unlikely to function in sister chromatid cohesion, since LBCs are post-replicative. Nevertheless, the rapid and specific accumulation of hRAD21-3HA on LBCs provides the opportunity to gain new insights into the mechanisms that regulate its chromosomal recruitment outside of S-phase.

The rapid recruitment of hRAD21-3HA to LBCs seems to require the chromomere-associated SMC1 and SMC3. Indeed, an in vitro made hRAD21 that is injected into oil-isolated nuclei associates with LBCs despite an apparent lack of free nucleoplasmic SMC1 and SMC3. One possibility is that chromomeric SMC1 and SMC3 serve as chromosomal docking sites for hRAD21-3HA. Alternatively, cohesin may diffuse off LBCs into the nucleoplasm, where RAD21 subunit exchange would occur prior to cohesin rebinding LBCs. Regardless of the mode of hRAD21 recruitment, it is likely to be directed to specific chromatin sites by SMC1 and SMC3 in the context of cohesin complexes. This conclusion is supported by our experiments, where hRAD21 deleted for either the SMC1 or SMC3 interacting domains fails to bind LBCs, or exhibits reduced binding, respectively.

The ability of hRAD21 deleted for its SMC3 interaction domain to bind LBCs, albeit at reduced levels, is surprising. Indeed, cohesin binding to chromosomes requires that all four cohesin subunits be functional (reviewed in Guacci 2007; Onn et al. 2008). In addition, there is no evidence that RAD 21 can bind chromosomes in the absence of SMC1 or SMC3. Cohesin was previously shown to form a tripartite ring with SMC1 and SMC3 dimerizing via their central hinge domains while RAD21 interacting with both SMCs to close the ring (Haering et al. 2002; Gruber et al. 2003). It is thought that the cohesin ring entraps both sisters chromatid during $\mathrm{S}$ phase to form cohesion (reviewed in Guacci 2007; Onn et al. 2008). Interestingly, the artificial cleavage of RAD21 in vivo resulted in cohesin dissociation from chromosomes in metaphase-arrested cells (Uhlmann et al. 2000). RAD21 lacking the SMC3 interacting domain should be unable to form a closed ring and, thus, unable to associate with chromosomes. It may be that cohesin can bind chromosomes in modes other than entrapment of chromatids within a ring. Supporting this idea is the finding that heterodimers of SMC1 and SMC3 exhibit weak DNA binding in vitro (Chiu et al. 2004). This mode of binding, independent from entrapment, could explain the results obtained on the LBC targeting of our hRAD21 mutants. Further experiments will be required to confirm this possibility, and whether this may represent an initial step in cohesin binding to chromosomes.

The idea that LBC-associated hRAD21-3HA is unlikely to function in sister chromatid cohesion is further supported by experiments using sperm heads, where single and unreplicated lampbrush chromatids rapidly acquire both SMC3 and hRAD21. Clearly, the cohesins associated with these newly formed chromatids are not involved in cohesion. Interestingly, the staining of hRAD21-3HA and endogenous SMC3 were consistently as strong on the lampbrush chromatids as on the LBCs, and more often stronger. Yet, the chromomeres of the unreplicated lampbrush chromatids are smaller, since they are composed of only half the DNA present in the LBC chromomeres (Gall and Murphy 1998). One interpretation at least for newly expressed hRAD21 is that most cohesin binding sites are already occupied on the LBC axes, while they are all available on the newly forming chromatids.

FRAP of cohesin in mammalian culture cells has recently provided valuable new insights in cohesin dynamics (Gerlich et al. 2006). There appear to be two distinct pools of cohesin in $\mathrm{G}_{2}$ phase cells discriminated by their residence time (slow and fast) on chromosomes. It was further proposed that since only the fast pool was detected in $\mathrm{G}_{1}$ phase cells, the more stably associated cohesin in $\mathrm{G}_{2}$ phase cells is the complex engaged in sister chromatid cohesion (Gerlich et al. 2006). Here, we demonstrate that chromosomal YFP-hRAD21-3HA is not locked on LBC chromomeres, but rather appears to exchange very rapidly with the nucleoplasm.

Newly made YFP-hRAD21-3HA, which is unlikely to act in cohesion, may corresponds to the more 
dynamic (fast) pool seen in $\mathrm{G}_{1}$ phase of mammalian cells. However, the recovery rate of YFP-hRAD213HA on LBCs is significantly faster than the $20 \mathrm{~min}$ rate observed for the fast pool in mammalian nuclei (Gerlich et al. 2006). There are a several possible explanations for this difference. First, our data reflects exchange kinetics of YFP-hRAD21-3HA at spatially defined chromosomal regions as opposed to measurements reflecting the combined recovery of chromosomally bound and free cohesin. Second, we examined meiotic cells where cohesin may need to be more dynamic to reflect chromosomal changes specific to meiosis. Third, the free nucleoplasmic pool of YFPhRAD21-3HA may be significantly higher in our experimental setting than the free pools of EGFPRAD21 or EGFP-SA1 used in mammalian cells (Gerlich et al. 2006). Finally, since the highly transcriptionally active lateral loops of LBCs emerge from chromomeres, rapid chromatin remodeling must be permitted to occur, which could justify in part the apparent high mobility of the cohesin there.

\section{Future perspectives}

Over the last decade, several experimental assays have been developed or optimized to study the structures and function of the various organelles present in the nuclei of amphibian oocytes. We present two of them here, namely the de novo formation of lampbrush chromatids (Gall and Murphy 1998) and the visualization of organelles (Handwerger et al. 2003), as well as LBCs in oil-isolated nuclei (Patel et al. 2008). The remarkable demonstration that sperm heads can yield individual lampbrush chromatids within a few hours after nuclear injection provides new opportunities of characterizing the dynamic molecular events that contribute to the formation and maintenance of LBCs. In particular, as new information is gained on the composition of the LBC chromatin, it will become possible to test the individual contribution of specific factors in a controlled and in vivo experimental assay. Since the examination of steady-state dynamics of LBC components is now possible, we can obtain valuable information about functional characteristics of chromatin factors that can only be hinted at using conventional nuclear spreads. For instance, defining and comparing the exchange kinetics of the various LBC-associated cohesin subunits would contribute to defining their interdependence for their recruitment to chromosomes. Furthermore, the characterization of various cohesin mutants can be used to provide insights into multiple aspects of cohesin function, including such as how cohesion establishment affects cohesin dynamics. Finally, methods to generate Xenopus frogs expressing a stably integrated transgene are readily available. We are currently engineering transgenic Xenopus frogs expressing YFP versions of different cohesin subunits. The goal will then be to use oocytes from these animals to investigate the distribution and dynamic properties of endogenous cohesins. Collectively, these various approaches represent original ways to investigate the extraordinary organization and plasticity of LBCs and fundamental aspects of chromatin.

Acknowledgements We thank Dr. T. Hirano (Cold Spring Harbor Laboratory) for his generous gift of the antibody directed against the amphibian SMC3. We are greatly indebted to Dr. J.G. Gall (Carnegie Institution of Washington) for providing the amazing images of LBC loops where the distribution patterns of histone H4 and RNAPII are compared. We would also like to thank him, as well as Dr. S.B. Patel, for very insightful discussions, and Dr. B. Beenders (UIUC) for the initial anti-cohesin antibody screen. We thank Dr. P.L. Jones (UIUC) for providing Xenopus sperm heads. Finally, we would like to thank our managing editor, Dr. Christian Haering, and the two anonymous reviewers for their very insightful and constructive critique of our manuscript. This work was a supported by a CAREER award from the National Science Foundation. Vincent Guacci is supported by the Howard Hughes Medical Institute.

\section{References}

Alexandru G, Uhlmann F, Mechtler K, Poupart MA, Nasmyth K (2001) Phosphorylation of the cohesin subunit Scc1 by Polo/Cdc5 kinase regulates sister chromatid separation in yeast. Cell 105:459-472

Beenders B, Watrin E, Legagneux V, Kireev I, Bellini M (2003) Distribution of XCAP-E and XCAP-D2 in the Xenopus oocyte nucleus. Chromosome Res 11:549-564

Beenders B, Jones PL, Bellini M (2007) The tripartite motif of nuclear factor 7 is required for its association with transcriptional units. Mol Cell Biol 27:2615-2624

Bellini M (2000) Coilin, more than a molecular marker of the cajal (coiled) body. Bioessays 22:861-867

Brar GA et al (2006) Rec8 phosphorylation and recombination promote the step-wise loss of cohesins in meiosis. Nature 441:532-536

Callan HG (1986) Lampbrush Chromosomes. Springer-Verlag, Berlin, Heidelberg, New York

Chiu A, Revenkova E, Jessberger R (2004) DNA interaction and dimerization of eukaryotic SMC hinge domains. J Biol Chem 279:26233-26242 
Ciosk R et al (1998) An ESP1/PDS1 complex regulates loss of sister chromatid cohesion at the metaphase to anaphase transition in yeast. Cell 93:1067-1076

Cohen-Fix O, Peters JM, Kirschner MW, Koshland D (1996) Anaphase initiation in Saccharomyces cerevisiae is controlled by the APC-dependent degradation of the anaphase inhibitor Pds1p. Genes Dev 10:3081-3093

Dorsett D (2007) Roles of the sister chromatid cohesion apparatus in gene expression, development, and human syndromes. Chromosoma 116:1-13

Fischer D, Hock R, Scheer U (1993) DNA topoisomerase II is not detectable on lampbrush chromosomes but enriched in the amplified nucleoli of Xenopus oocytes. Exp Cell Res 209:255-260

Flemming W (1882) Zellsubstanz, Kern und Zelltheilung. F. C. W. Vogel, Leipzig

Funabiki H, Kumada K, Yanagida M (1996) Fission yeast Cut1 and Cut2 are essential for sister chromatid separation, concentrate along the metaphase spindle and form large complexes. EMBO J 15:6617-6628

Gall JG (1956) On the submicroscopic structure of chromosomes. In Mutation 8:17-32

Gall JG, Murphy C (1998) Assembly of lampbrush chromosomes from sperm chromatin. Mol Biol Cell 9:733-747

Gall JG, Bellini M, Wu Z, Murphy C (1999) Assembly of the nuclear transcription and processing machinery: Cajal bodies (coiled bodies) and transcriptosomes. Mol Biol Cell 10:4385-4402

Gerlich D, Koch B, Dupeux F, Peters JM, Ellenberg J (2006) Live-cell imaging reveals a stable cohesin-chromatin interaction after but not before DNA replication. Curr Biol 16:1571-1578

Glynn EF et al (2004) Genome-wide mapping of the cohesin complex in the yeast Saccharomyces cerevisiae. PLoS Biol 2:E259

Gruber S, Haering CH, Nasmyth K (2003) Chromosomal cohesin forms a ring. Cell 112:765-777

Guacci V (2007) Sister chromatid cohesion: the cohesin cleavage model does not ring true. Genes Cells 12:693-708

Guacci V, Hogan E, Koshland D (1994) Chromosome condensation and sister chromatid pairing in budding yeast. J Cell Biol 125:517-530

Guacci V, Koshland D, Strunnikov A (1997) A direct link between sister chromatid cohesion and chromosome condensation revealed through the analysis of MCD1 in S. cerevisiae. Cell 91:47-57

Gurdon JB, De Robertis EM, Partington G (1976) Injected nuclei in frog oocytes provide a living cell system for the study of transcriptional control. Nature 260:116-120

Haering CH et al (2004) Structure and stability of cohesin's Smc1-kleisin interaction. Mol Cell 15:951-64

Haering CH, Lowe J, Hochwagen A, Nasmyth K (2002) Molecular architecture of SMC proteins and the yeast cohesin complex. Mol Cell 9:773-788

Handwerger KE, Murphy C, Gall JG (2003) Steady-state dynamics of Cajal body components in the Xenopus germinal vesicle. J Cell Biol 160:495-504

Hirano T, Kobayashi R, Hirano M (1997) Condensins, chromosome condensation protein complexes containing XCAP-C, XCAP-E and a Xenopus homolog of the Drosophila Barren protein. Cell 89:511-521
Hock R, Moorman A, Fischer D, Scheer U (1993) Absence of somatic histone $\mathrm{H} 1$ in oocytes and preblastula embryos of Xenopus laevis. Dev Biol 158:510-522

Jessberger R (2002) The many functions of SMC proteins in chromosome dynamics. Nat Rev Mol Cell Biol 3:767-778

Jessberger R (2003) SMC proteins at the crossroads of diverse chromosomal processes. IUBMB Life 55:643-652

Kateneva AV, Konovchenko AA, Guacci V, Dresser ME (2005) Recombination protein Tid1p controls resolution of cohesindependent linkages in meiosis in Saccharomyces cerevisiae. J Cell Biol 171:241-253

Klein F et al (1999) A central role for cohesins in sister chromatid cohesion, formation of axial elements, and recombination during yeast meiosis. Cell 98:91-103

Krasikova A, Barbero JL, Gaginskaya E (2005) Cohesion proteins are present in centromere protein bodies associated with avian lampbrush chromosomes. Chromosome Res 13:675-685

Krasikova A et al (2006) On the positions of centromeres in chicken lampbrush chromosomes. Chromosome Res 14:777-789

Kumada K et al (1998) Cut1 is loaded onto the spindle by binding to Cut2 and promotes anaphase spindle movement upon Cut2 proteolysis. Curr Biol 8:633-641

Leon P, Kezer J (1990) Loop size in newt lampbrush chromosomes. Chromosoma 99:83-86

Losada A, Hirano M, Hirano T (1998) Identification of Xenopus SMC protein complexes required for sister chromatid cohesion. Genes Dev 12:1986-1997

Losada A, Yokochi T, Kobayashi R, Hirano T (2000) Identification and characterization of $\mathrm{SA} / \mathrm{Scc} 3 \mathrm{p}$ subunits in the Xenopus and human cohesin complexes. J Cell Biol 150:405-416

Lund E, Paine PL (1990) Nonaqueous isolation of transcriptionally active nuclei from Xenopus oocytes. Methods Enzymol 181:36-43

McKay MJ et al (1996) Sequence conservation of the $\operatorname{rad} 21$ Schizosaccharomyces pombe DNA double-strand break repair gene in human and mouse. Genomics 36:305-315

McNairn and Gerton, this issue

Michaelis C, Ciosk R, Nasmyth K (1997) Cohesins: chromosomal proteins that prevent premature separation of sister chromatids. Cell 91:35-45

Morgan GT (2002) Lampbrush chromosomes and associated bodies: new insights into principles of nuclear structure and function. chromosome Res 10:177-200

Murphy C, Wang Z, Roeder RG, Gall JG (2002) RNA polymerase III in Cajal bodies and lampbrush chromosomes of the Xenopus oocyte nucleus. Mol Biol Cell 13:3466-3476

Newmeyer DD, Wilson KL (1991) Egg extracts for nuclear import and nuclear assembly reactions. In: Kay BK, Peng HB (eds) Xenopus laevis: Practical Uses in Cell and Molecular Biology, Vol. 36. Academic Press, San Diego, pp 607-634

Onn I, Heidinger-Pauli JM, Guacci V, Unal E, Koshland DE (2008) Sister chromatid cohesion: a simple concept with a complex reality. Annu Rev Cell Dev Biol

Paine PL, Johnson ME, Lau Y-T, Tluczek LJM, Miller DS (1992) The oocyte nucleus isolated in oil retains in vivo structure and functions. BioTechniques 13:238-245 
Parisi S et al (1999) Rec8p, a meiotic recombination and sister chromatid cohesion phosphoprotein of the Rad21p family conserved from fission yeast to humans. Mol Cell Biol $19: 3515-3528$

Parra MT et al (2004) Involvement of the cohesin Rad21 and SCP3 in monopolar attachment of sister kinetochores during mouse meiosis I. J Cell Sci 117:1221-1234

Patel SB, Novikova N, Bellini M (2007) Splicing-independent recruitment of spliceosomal small nuclear RNPs to nascent RNA polymerase II transcripts. J Cell Biol 178:937-949

Patel S, Novikova N, Beenders B, Austin C, Bellini M (2008) Live images of RNA polymerase II transcription units. Chromosome Res 16:223-232

Prieto I et al (2004) Cohesin component dynamics during meiotic prophase I in mammalian oocytes. Chromosome Res 12:197-213

Revenkova E, Jessberger R (2005) Keeping sister chromatids together: cohesins in meiosis. Reproduction 130:783-790

Rückert J (1892) Zur Entwickelungsgeschichte des Ovarialeies bei Selachiern. Anatomische Anzeiger 7:107-158

Sommerville J, Baird J, Turner BM (1993) Histone H4 acetylation and transcription in amphibian chromatin. $\mathrm{J}$ Cell Biol 120:277-290

Stewart MD, Sommerville J, Wong J (2006) Dynamic regulation of histone modifications in Xenopus oocytes through histone exchange. Mol Cell Biol 26:6890-6901

Stoop-Myer C, Amon A (1999) Meiosis: Rec8 is the reason for cohesion. Nat Cell Biol 1:E125-127
Sumara I, Vorlaufer E, Gieffers C, Peters BH, Peters JM (2000) Characterization of vertebrate cohesin complexes and their regulation in prophase. J Cell Biol 151:749-762

Toth A et al (1999) Yeast cohesin complex requires a conserved protein, Ecolp(Ctf7), to establish cohesion between sister chromatids during DNA replication. Genes Dev 13:320-333

Tuma R, Stolk JA, Roth MB (1993) Identification and characterization of a sphere organelle protein. J Cell Biol 122:767-773

Uhlmann F, Lottspeich F, Nasmyth K (1999) Sister-chromatid separation at anaphase onset is promoted by cleavage of the cohesin subunit Scc1. Nature 400:37-42

Uhlmann F, Wernic D, Poupart MA, Koonin EV, Nasmyth K (2000) Cleavage of cohesin by the CD clan protease separin triggers anaphase in yeast. Cell 103:375-386

Waizenegger IC, Hauf S, Meinke A, Peters JM (2000) Two distinct pathways remove mammalian cohesin from chromosome arms in prophase and from centromeres in anaphase. Cell 103:399-410

Wallace RA, Jared DW, Dumont JN, Sega MW (1973) Protein incorporation by isolated amphibian oocytes: III. Optimum incubation conditions. J Exp Zool 184:321-333

$\mathrm{Xu} \mathrm{H}$ et al (2004) A new role for the mitotic RAD21/SCC1 cohesin in meiotic chromosome cohesion and segregation in the mouse. EMBO Rep 5:378-384

Xu H, Beasley MD, Warren WD, van der Horst GT, McKay MJ (2005) Absence of mouse REC8 cohesin promotes synapsis of sister chromatids in meiosis. Dev Cell 8:949-61 